\title{
Phytophthora capsici PcFtsZ2 Is Required for Asexual Development and Plant Infection
}

\author{
Jing $\mathrm{Li},{ }^{1}$ CongCong Ai, ${ }^{1}$ CanCan Yang, ${ }^{1}$ WeiLin Jiang, ${ }^{1}$ Hui Sheng, ${ }^{1}$ Paul F. Morris, ${ }^{2}$ and \\ XiuGuo Zhang ${ }^{1,+}$ \\ ${ }^{1}$ Shandong Provincial Key Laboratory for Biology of Vegetable Diseases and Insect Pests, College of Plant Protection, \\ Shandong Agricultural University, Tai'an, 271018, China \\ ${ }^{2}$ Department of Biological Sciences, Bowling Green State University, Bowling Green, OH 43403, U.S.A.
}

Accepted 24 February 2020. Retracted 28 November 2022.

In bacteria, FtsZ proteins form a $\mathrm{Z}$ ring that is the initial step preceding septal fission. FtsZ proteins enable the division of mitochondria in early eukaryotes and are present in some kingdoms but have been lost in animals, fungi, and plants. Here, we have identified two Phytophthora capsici ortholog genes of Escherichia coli FtsZs, designated PcFtsZ1 and PcFtsZ2. Overexpression of PcFtsZ2 in E. coli fully complemented the overexpression phenotype of EcFtsZ. In contrast, overexpression of PcFtsZ1 in E. coli had minimal impact on cell division and separation. Thus, we focused on evaluating the impact of altered expression of PcFtsZ2 in P. capsici, as it exhibited the strongest phenotype. PcFtsZ2 was expressed at the highest levels in mycelia, sporangia, and germinating cysts, as well as in late infection. PcFtsZ2 mis-expression lines showed aberrant asexual growth and development of $P$. capsici. Alterations in the expression of PcFtsZ2 changed the distribution of mitochondria in hyphae and sporangia and, also, affected the number, size, and shape of actin plaques. Silencing of PcFtsZ2 restrained growth and development of invasive structures, especially cysts and sporangia, substantially inhibiting the ability of transformants to cause blight lesions. In overexpressed transformant lines, cyst and sporangial germination rates were only half that of controls, but hyphal growth from direct germination of sporangia was more rapid than controls. These transformant lines were only slightly impaired in virulence relative to controls. This study emphasizes the essential role of the evolutionarily conserved FtsZ2 proteins in affecting cytoskeleton dynamics.

Keywords: FtsZ proteins, oomycete-plant interactions

Despite the genomic and morphological diversity of eukaryotic cells, all these cells share a number of features in common. They include a nucleus and multiple organelles as

${ }^{\dagger}$ Corresponding author: X. Zhang; zhxg@sdau.edu.cn

Funding: This work was supported by the Special Fund for Agro-scientific Research in the Public Interest of China (2017YFD0200600, 2017YFD0201601, 2017YFD0200901, 201003004).

*The $\boldsymbol{e}$-Xtra logo stands for "electronic extra" and indicates that 12 supplementary figures and two supplementary tables are published online.

The author(s) declare no conflict of interest.

๑) 2020 The American Phytopathological Society well as numerous proteins that are shared across all kingdoms. All these proteins are referred to as eukaryotic signature proteins, strongly suggesting that they are also present in the last eukaryotic common ancestor. These conserved proteins are involved in DNA and RNA synthesis, endosomal sorting, vesicle trafficking, cytoskeletal organization, and cell division. Some of these proteins have recently been found in reconstructed Asgard genomes, indicating that these eukaryoticspecific proteins are already present in the group of archaea (Zaremba-Niedzwiedzka et al. 2017).

Oomycete plant pathogens damage a wide range of dicotyledonous plants. These include the potato late blight pathogen Phytophthora infestans as well as P. capsici, which attacks a number of cucurbitaceae species (Erwin and Ribeiro 1996; Lamour et al. 2012). The oomycetes are grouped in the heterokonts, which include photosynthetic diatoms (Baldauf et al. 2000). Oomycetes grow as mycelium but also produce asexual spores that serve as dispersal agents and infect host tissues (Judelson and Blanco 2005; Latijnhouwers et al. 2003; Randall et al. 2005; Walker and van West 2007). Cytoskeletal proteins play important roles in the formation of the structures that are necessary for infection and reproduction. The actin cytoskeleton is an integral part of all eukaryotic cells and has been the most extensively studied cytoskeleton component in oomycetes (Bachewich and Heath 1998; Ketelaar et al. 2012; Kots et al. 2017; Meijer et al. 2014). In oomycetes, actin is organized as actin cables or as large structures termed actin plaques (Deora et al. 2008; Meijer et al. 2014). These plaques are associated with quiescent regions in the cell (Ketelaar et al. 2012). Moreover, actin organization is essential for the formation of special infection structures such as appressorium, cell plug, and the hyphal tip (Kots et al. 2017; Temperli et al. 1990).

Other cytoskeletal components that are thought to be present in the last eukaryotic common ancestor including dynamins (Purkanti and Thattai 2015) and FtsZ proteins (Leger et al. 2015). In bacteria, FtsZ acts in concert with MinC, MinD, and MinE, which are septum site-determining proteins, to constrict the cell membrane at the site of cell fission (Colletti et al. 2000; Gregory et al. 2008; Hu et al. 1999; Johnson et al. 2002; Levin et al. 1998). Fts $Z$ polymerizes to form a ring structure (the $Z$ ring) under the membrane at a site determined by the Min proteins. The $\mathrm{Z}$ ring constricts to initiate division and remains at the leading edge of the invaginating septum during cell division (Anderson et al. 2004; Vitha et al. 2001; Xiao and Goley 2016).

Homologs of the FtsZ-associated proteins MinC, MinD, and MinE had also been recognized in the genomes of diverse eukaryotes (Osteryoung et al. 1998; Turmel et al. 1999). In photosynthetic eukaryotes, FtsZ1 and FtsZ2 form a Z ring on the 
inside of the chloroplast membrane, and knockouts of either gene prevents chloroplast division (TerBush et al. 2013). MinD and MinE are retained in plant lineages, but MinC has been lost and replaced by ARC3 in many species (Chen et al. 2018).

In plants, animals, and fungi, mitochondrial FtsZ and MinC, Min D, and MinE have been lost, and mitochondrial division is facilitated by dynamins that form an external contractile ring around the dividing organelle (Arimura and Tsutsumi 2002; Beech et al. 2000; Moore and Holzbaur 2018). In other eukaryotic kingdoms, including Amebozoa, Stramenopila, Rhodophyta, and Excavata, FtsZ and MinC, MinD, and MinE have been retained in many species that have been investigated (Baldauf et al. 2000). These organisms have been postulated to be 'living fossils' containing a cytoskeletal network utilizing both dynamins, FtsZ proteins and MinC, MinD, and MinE (Kiessling et al. 2000; TerBush et al. 2013). FtsZs have been retained in eukaryotes, and two distinct isoforms are present in each genome (Araki et al. 2003; Miyagishima et al. 2004; Stokes and Osteryoung 2003). The two isoforms form two distinct clades, FtsZ1 and FtsZ2, that span the lineages and are involved in mitochondria and plastids (Gilson and Beech 2001), suggesting that there is an ancient duplication of the FtsZ gene in a common ancestor of these lineages (Osteryoung and McAndrew 2001). Red algae and photosynthetic stramenopiles contain only the FtsZ1 clade (Matsuzaki et al. 2004). Constituents of the FtsZ2 clade are characterized by a variable Cterminal spacer region containing at least a portion of the sequence (D/E) (I/V) PX(F/Y) L (Din et al. 1998; Ma and Margolin 1999).

The functions of eukaryotic FtsZ proteins have only been characterized in the amebozoa Dictyostelium and the photosynthetic stramenopile Mallamonas. Colocalization of MitoTracker Green and antibody-labeled Mallamonas splendens cells indicated that FtsZ proteins were localized to the mitochondria. Transformation of green fluorescent protein (GFP)labeled MsFtsZ proteins in yeast also resulted in localization of the proteins to mitochondria. The functions of both $F t s Z$ genes (FtsZ2 homolog FtsZA and FtsZ1 homolog FtsZB) of Dictyostelium discoideum were investigated (Gilson et al. 2003), and GFP fusions revealed that the two proteins had different locations in the mitochondria. FtsZA formed belts and puncta in the mitochondria, whereas FtsZB concentrated in submitochondrial body. Knockout of either of the genes caused malformation and reduced numbers of mitochondria. However, the double knockout did not show a stronger phenotype than either single knockout, suggesting that each protein makes an essential contribution to the same process but is not functionally redundant. The effect of FtsZA protein expression was evaluated by looking at cell lines with different fluorescence intensity of FtsZA-GFP. At lower expression levels, FtsZA formed belts and puncta, recognized as a localization pattern in spherical mitochondria. At higher expression levels, mitochondria appeared tubular, suggesting an inhibition of mitochondrial division, and FtsZA-GFP formed helices with the mitochondria. The FtsZ proteins appeared to have separate localizations within the mitochondria of Dictyostelium discoideum, while, in the chloroplast of Arabidopsis thaliana, FtsZ1 and FtsZ2 are associated with the Z ring as homopolymers, forming a protein complex tying the $\mathrm{Z}$ ring to the membrane and connecting to a dynamin-type protein surrounding the chloroplast on the outside (Chen et al. 2018).

The roles of FtsZ proteins have not been examined in oomycetes, in which the positioning of mitochondria and regulated mitochondrial division might be expected to be critical for organisms that have large multinucleated cells, polarized growth, cytoplasmic migration, and a variety of developmental cell types. Here, we characterize the functions of FtsZ1 and
FtsZ2 in Escherichia coli and FtsZ2 in P. capsici. Overexpression of PcFtsZ2 fully complements the overexpression phenotype of EcFtsZ, indicating the domains needed for cellular fission are retained. In contrast, cultures of $E$. coli overexpressing PcFtsZ1 had fewer examples of short chains of cells. Transformants of $P$ capsici that had reduced or overexpressed levels of PcFtsZ2 displayed impairments in growth and development and a reduction in the ability to cause disease. These phenotypes were also associated with substantive changes in the distribution of filamentous actin and actin plaques. This study demonstrates the essential role of the evolutionarily conserved FtsZ2 proteins in affecting cytoskeleton dynamics.

\section{RESULTS}

\section{Identification of FtsZ homologs}

in the genome sequence of $P$. capsici.

A FtsZ sequence of $P$. capsici was retrieved using the fulllength sequence of the E. coli FtsZ proteins, by means of the program TBLASTN, with an expected (E) value cut-off $<10^{-15}$. Initially, only a single gene model (estExt2_Genewise1.C_ PHYCAscaffold_330142) was detected, which encoded a protein having $30 \%$ amino acid identity with E. coli FtsZ. This gene contained a predicted mitochondrial transit peptide (Supplementary Figs. S1 and S2) and encoded a protein that was highly conseryed from end to end with other Phytophthora FtsZ homologs and closely matched other eukaryotic FtsZ2 sequences. Thus, we named this gene PcFtsZ2. PcFtsZ2 was cloned from $P$. capsici (SD33) DNA using primers (Supplementary Table S1). The sequence has been deposited in GenBank (number AKB91760.1) (Supplementary Table S2).

Other sequenced oomycete genomes contain two FtsZ genes (Tyler et al. 2006), and closer examination of the genome of $P$. capsici identified another gene model spanning nine introns, which corresponded to gene model fgenesh2_kg.PHYCAscaffold_27_\#_83_\#_4098303, except that this model lacked the codons encoding the first four residues (MAIS) that are conserved in $P$. parasitica and $P$. infestans homologs (Supplementary Fig. S3). This FtsZ gene encoded a protein that most closely matched eukaryotic FtsZ1 sequences, and this gene was named PcFtsZ1. PcFtsZ1 was cloned from P. capsici (SD33) DNA using primers (Supplementary Table S1). The sequence has been deposited in GenBank (number MN764110) (Supplementary Table S2). PcFtsZ1 and PcFtsZ2 encode 418 and 472 amino acids, respectively, with calculated molecular masses of 43.7 and $50.2 \mathrm{kDa}$, including predicted mitochondrial transit peptides. Relative to most other Phytophthora proteins, PcFtsZ1 and PcFtsZ2 proteins are strongly conserved. PcFtsZ1 shares $93.3 \%$ identity with FtsZ1 in P. infestans and 92.4 and $91.7 \%$ with its orthologs in $P$. parasitica and $P$. sojae, respectively. Similarly, PcFtsZ2 shares $88 \%$ identity with its ortholog in P. infestans and 89.3 and $85.5 \%$ with its orthologs in $P$. parasitica and $P$. sojae, respectively (Supplementary Fig. S3; Supplementary Table S2).

PcFtsZ1 and PcFtsZ2 share only $38.1 \%$ identity with each other overall, but within a central conserved core (residues of 109 to 304 in PcFtsZ1 and 116 to 303 in PcFtsZ2), the identity is $53.0 \%$. In contrast, the central core of FtsZ1 and FtsZ2 in A. thaliana is $61 \%$ identical over a span of 308 residues. Thus, the oomycete proteins are much more divergent from each other than FtsZ1 and FTsZ2 from A. thaliana.

The core region of oomycete proteins showed similar levels of identity with E. coli FtsZ (51.3 and $45.5 \%$, respectively), and higher levels of similarity with FtsZ sequences from $\alpha$-proteobacteria such as BAL31 (63.8 and $58.7 \%$, respectively) and Rickettsia tamurae (61.2 and 56.6\%) (Supplementary Fig. S4). 
These patterns of conservation are consistent with the ancient origin of the split between eukaryotic FtsZ1 and FtsZ2 sequences, and the $\alpha$-proteobacterial origin of mitochondria (Esser et al. 2004).

The Phytophthora FtsZs also contained three conserved motifs, a typical GTP-binding motif (GMGGGTGTG) and two other conserved motifs, GG $/{ }_{\mathrm{G}} \mathrm{GXNAV} \times \mathrm{xM}$ and PGLINLDFAD (Kiefel et al. 2004), which are well-conserved in most FtsZs (Supplementary Fig. S4). The bacterial FtsZs C-termini display highly conserved IPAFLR motifs (Din et al. 1998; Ma and Margolin 1999), while all the Phytophthora FtsZ2s contain a fully conserved GLFGRFFSL motif in the corresponding region. This motif conforms to $\mathrm{G} / \mathrm{L} / \mathrm{F} / \mathrm{G} / \mathrm{S} / \mathrm{R} / \mathrm{F} / \mathrm{F}_{\mathrm{X}} \mathrm{S} / \mathrm{L}$ sequence that is partially conserved in another oomycete, FtsZ2s, and bears a slight similarity to the bacterial motif. The FtsZ1s entirely lack the variable tail, like other eukaryotic FtsZ1s.

The predicted amino acid sequences of the 47 putative FtsZ genes from plants, eubacteria, archaea, and oomycetes (Supplementary Table S2) were aligned using Clustal X (Thompson et al. 1997). Phylogenetic analyses were performed on MEGA5 by the neighbor-joining method. Phylogenetic trees were constructed using alignment of approximately 300 conserved amino acids of these selected FtsZs. A phylogenetic tree is shown in Supplementary Figure S5. The branches in the tree were identified as three monophyletic groups, such as Phytophthora bacteria, plant, and archaea, which were similar to previous observations (Beech et al. 2000; Erickson 1997; Kiefel et al. 2004; Takahara et al. 2000). In this tree, two PcFtsZs and other selected Phytophthora FtsZs were unequivocally clustered with FtsZ proteins from other eubacteria; thus, these Phytophthora FtsZs and other procaryotic FtsZ genes are predicted to have the same ancestral origins. Thus, we clearly believe that PcFtsZs have the possibility to modulate asexual growth and development.
Functional expression of PcFtsZ1 and PcFtsZ2 in E. coli.

In bacteria, Fts $\mathrm{Z}$ proteins form the $\mathrm{Z}$ ring around the cell, which then constricts to initiate the process of cell division (Bi and Lutkenhaus 1991; Erickson et al. 2010). However, high expression levels of the FtsZ genes are related to an inhibition of cellular division (Ward and Lutkenhaus 1985). To determine whether PcFtsZ1 and PcFtsZ2 were able to partially complement the functions of EcFtsZ, we expressed PcFtsZ1, PcFtsZ2, EcFtsZ, or an empty vector control (CK) in E. coli JM109 (wild type [WT]). Endogenous expression of EcFtsZ protein (44 kDa) was shown in the lower bands of all cell lines on sodium dodecyl sulfate-polyacrylamide gel electrophoresis (SDSPAGE) gels (Fig. 1A), and increased expression of a 54-kDa band was shown, in the upper band of the PcFtsZ2 line, that was close to the size of the expected PcFtsZ2 protein. The PcFtsZ1 protein expression in E. coli JM109 was not shown in this study. Protein expression levels of PcFtsZ2 and EcFtsZ (Fig. 1A) and PcFtsZ1 in all cell lines were verified by Western blot using anti-histidine (His) antibody (Supplementary Fig. S7D). The size of the PcFtsZ1 protein was expected to be $46 \mathrm{kDa}$. As expected, CK and WT controls did not express these target bands (Fig. 1A; Supplementary Fig. S7D).

The cell morphology of the recombinant strains PcFtsZ1, PcFtsZ2, and EcFtsZ was compared with the two controls strains by light microscopy. Cells of the two controls showed normal morphology of the E. coli. Cultures of PcFtsZ2 and EcFtsZ strains contained some elongated cells with partial constrictions, and many examples of partially separated cells that formed short or long filament chains (Fig. 1B; Supplementary Fig S7A). Thus, PcFtsZ2 fully complemented the overexpression phenotype of EcFtsZ (Ward and Lutkenhaus 1985). In contrast, cultures of PcFtsZ1 contained only a few examples of short chains of cells and overexpression of this gene had minimal effects on bacterial division.
A

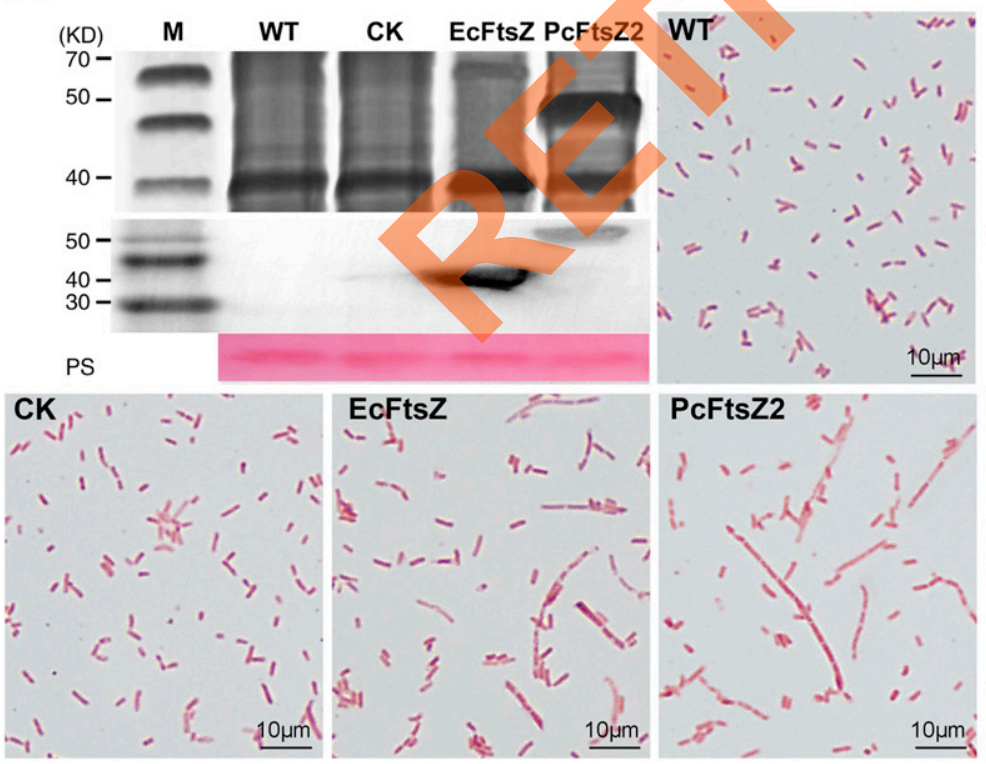

C
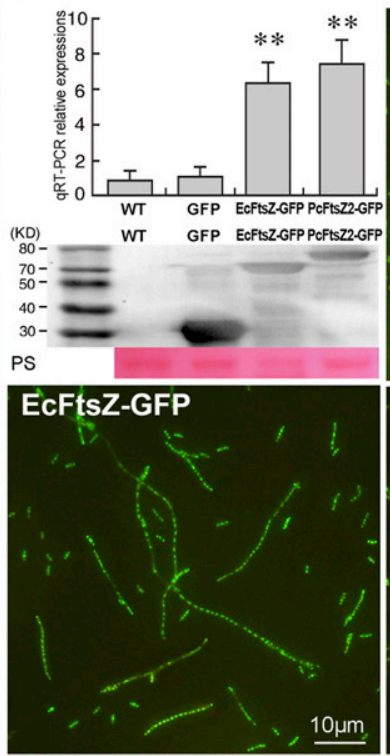

D
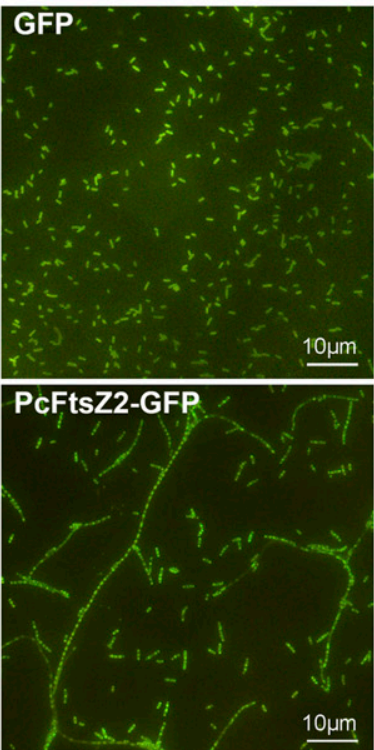

Fig. 1. Morphology of Escherichia coli is affected by heterologous expression of PcFtsZ2. A, Sodium dodecyl sulfate-polyacrylamide gel electrophoresis (upper) and Western blot (below) analysis of pET28a-PcFtsZ2 and pET28a-EcFtsZ expression in E. coli JM109. B, Cellular morphology of E. coli JM109 in the recombinant lines, wild type (WT) (E. coli JM109), CK (pET28a vector line), EcFtsZ (pET28a-EcFtsZ line), and PcFtsZ2 (pET28a-PcFtsZ2 line). C, Transcript levels of PcFtsZ2-GFP (green fluorescent protein) and EcFtsZ-GFP in different recombinant transformants were analyzed by quantitative reverse transcription-PCR. Transcript levels of EcFtsZ in E. coli cells were used to contrast the expression levels of PcFtsZ2 or EcFtsZ in different transformants. Western blots reveal that EcFtsZ-GFP or PcFtsZ2-GFP protein is intact and stable in different transformants using GFP antibody. PS = Ponceau staining. D, Cellular morphology of $E$. coli JM109 in different transformants. Three replicates for each sample were used to calculate standard errors. In C and D, WT $=E$. coli JM109, GFP = pEHIS-GFP-TEV-1, EcFtsZ-GFP = pEHIS-GFP-TEV-EcFtsZ-1, and PcFtsZ2-GFP = pEHIS-GFP-TEV-PcFtsZ2-1. 
To localize PcFtsZ1, PcFtsZ2, and EcFtsZ in E. coli cells, we generated the transformants of GFP-PcFtsZ1, GFP-PcFtsZ2, GFP-EcFtsZ, and CK (pEHIS-GFP-TEV [tobacco etch virus] was transformed into E. coli JM109). Four, four, five, and three stable transformants of these constructs were obtained, respectively. The transcript levels of PcFtsZ1, PcFtsZ2, and EcFtsZ in GFP-PcFtsZ1, GFP-PcFtsZ2, or GFP-EcFtsZ were significantly elevated compared with the two controls (Fig. $1 \mathrm{C}$; Supplementary Fig. S7C). In fact, PcFtsZ1 and PcFtsZ2 transcript levels in the GFP-PcFtsZ1 and GFP-PcFtsZ2 lines were not significantly different from each other, and a similar uniform pattern of expression of GFP-EcFts $Z$ was observed in five GFP-EcFtsZ lines. Thus, a single transformant of each type was selected for comparative analysis. The endogenous EcFtsZ transcript levels were used to compare those of PcFtsZ1 and PcFtsZ2 in the different transformants (Fig. 1C; Supplementary Fig. S7C). Thus, the ratio between the plasmid-derived and endogenous transcripts could be directly obtained.

We used a GFP-antibody to confirm the expression of PcFtsZ1-GFP, PcFtsZ2-GFP, and EcFtsZ-GFP proteins, singly or in combination, in JM109. The PcFtsZ2-GFP, PcFtsZ1-GFP, and EcFtsZ-GFP proteins are approximately 80, 76, and $70 \mathrm{kDa}$ respectively. The E. coli JM109 cells did not produce a detectable GFP fusion protein (Fig. 1C; Supplementary Fig. S7E). The GFP protein was used as negative control. Analysis of Western blots indicated that PcFtsZ1-GFP, PcFtsZ2-GFP, EcFtsZ-GFP, and GFP fusion proteins were expressed at similar levels in the different transformants. The cells of the GFP control strain showed a bacilliform shape (Fig. 1D, Supplementary Fig. S7B), similar those shown in Figure 1B and Supplementary Figure S7A, which were the normal morphology of the $E$. coli cells. In contrast, filaments and chains of cells were observed in GFP-EcFtsZ and GFP-PcFtsZ2 (Fig. 1D) similar to those shown in Figure 1B. The distribution of GFP fluorescence in EcFTsZ-GFP, PcFtsZ1-GFP, or PcFtsZ2-GFP overexpression strains was highly punctate, with the GFP fluorescence appearing at highly regularly spaced intervals, as previously described (Ma et al. 1996). To summarize, while FtsZ1 had a similar localization pattern as FtsZ2 and EcFtsZ, its expression only slightly inhibited bacterial fission and separation.

\section{PcFtsZ2 showed differential expression}

\section{during development and infection stage}

Since PcFtsZ1 and PcFtsZ2 proteins are highly divergent from each other, and thus likely have separate but conserved roles across Phytophthora spp., this study focused on a functional analysis of PcFtsZ2. We first measured the expression patterns of PcFtZ2, by quantitative reverse transcription PCR (qRT-PCR), in mycelia, sporangia, zoospores, cysts, and germinating cysts (Supplementary Fig. S6). Expression levels in the mycelia, sporangia, and germinating cyst stages were about three-fold higher than in zoospores and cysts $(P<0.01$; Wilcoxon rank sum test). PcFtZ2 showed the lowest expression levels in zoospores and cysts (23 and $30 \%$ of mycelia, both $P<0.05$ ). During infection, expression levels increased with time, peaking at $24 \mathrm{~h}$ when expression was almost threefold higher than at $1.5 \mathrm{~h}$. Expression then declined from peak levels over the next 2 days. To summarize, PcFtsZ2 is expressed at all developmental stages as well as during infection, but expression is the lowest in zoospores and cysts. Expression levels of PcFtsZ2 appear to be the highest in tissues associated with high rates of cellular division (mycelia, developing sporangia, and infected tissues).

\section{Generation of PcFtsZ2-silenced and} overexpression transformants in $P$. capsici.

To test the functions of PcFtsZ2 in P. capsici, we created PcFtsZ2 silencing and overexpression transformants. PcFtsZ2 transcript levels were substantially depressed in five transformants (SiPcFtsZ2-1, SiPcFtsZ2-2, SiPcFtsZ2-3, SiPcFtsZ24, and SiPcFtsZ2-5), as determined by RT-PCR (data not shown) and qRT-PCR assays. In the silenced lines, the expression of PcFtsZ2 was reduced about eightfold $(P<0.01)$ relative to WT and CK strains (Fig. 2B; Supplementary Figs. S8B and S9A). Each of the silenced lines exhibited the same abnormalities in hyphal growth and sporangial development relative to WT and CK (data not shown). Thus, the phenotypes of three typically silenced lines (SiPcFtsZ2-1, SiPcFtsZ2-2, and SiPcFtsZ2-3) were used for comparative studies. The phenotypes of SiPcFtsZ2-1 and SiPcFtsZ2-2 are shown in Figure 2A, while those of SiPcFtsZ2-3 are shown in Supplementary Figure S8A. In a similar fashion, the overexpression of PcFtsZ2 was confirmed in five overexpression lines (OPcFtsZ2-1, OPcFtsZ2-2, OPcFtsZ2-3, OPcFtsZ2-4, and OPcFtsZ2-5). In the overexpression lines, expression levels were increased about 7.5 -fold $(P<0.01)$ relative to two controls (Figs. 2B; Supplementary Figs. S8C and S9B). Since the phenotypes of these overexpression lines were similar, three lines (OPCFtsZ21, OPcFtsZ2-2, and OPcFtsZ2-3) were selected for comparison purposes. The characteristic phenotypes of OPcFtsZ2-1 and OPcFtsZ2-2 are shown in Figure 2A and that of OPcFtsZ2-3 is presented in Supplementary Figure S8A.

\section{Influence of PcFtsZ2 on hyphal growth and the number and size of sporangia.}

Next, we examined the impact of alterations on the expression of PcFtsZ2 when transformants were grown on V8 agar plates. The growth rate of three silenced transformants was significantly less than in three overexpression lines $(P<0.05)$ and $\mathrm{CK}$ and WT controls $(P<0.01)$ (Fig. $2 \mathrm{~A}$ and $\mathrm{C}$; Supplementary Fig. S8A and D; Table 1). The growth rate of the overexpression lines was greater than silenced lines but also significantly less than controls $(P<0.05)$. As illustrated in Figure 2A and Supplementary Figures S8A and S10A and B, the colonies of SiPcFtsZ2-1, SiPcFtsZ-2, and SiPcFtsZ-3 formed a dense hyphal mass and lacked aerial hyphae, while the colonies of OPcFtsZ2-1, OPcFtsZ2-2, and OPcFtsZ2-3 produced a less dense hyphal mat with abundant aerial hyphae. Therefore, normal regulation of PcFtsZ2 is essential for mycelial growth of $P$. capsici.

Expression levels of PcFtsZ2 influenced the number and size of sporangia in different transformants. As shown in Figure 2A, Supplementary Figure S8A, and Table1, silenced or overexpression of PcFtsZ2 significantly influenced the number of sporangia in three silenced lines $(P<0.01)$ and the three overexpression lines $(P<0.05)$ when compared with the two controls. In overexpression lines, sporangial density was approximately $60 \%$ of controls, while, in silenced lines, sporangial density was approximately $30 \%$ of controls. As shown in Figure 2A and D and Supplementary Figure S8A and E, PcFtsZ2 expression also influenced the size of sporangia in three silenced lines $(P<0.01)$ and three overexpression lines $(P<0.05)$ when compared with the two controls. Thus, we conclude that PcFtsZ2 is necessary for normal sporangial development.

\section{Influence of PcFtsZ2 on sporangiophore development and sporangial morphology.}

In cultures of $P$. capsici, aerial hyphae form sporangiophores that then produce multiple branches, and a number of sporangia develop at the tip of each branch (Lamour et al. 2012). The sporangia are typically ovoidal to fusiform and develop into a single terminal papilla. To evaluate how the growth of sporangiophore and development of sporangial shape were influenced by PcFtsZ2, we first imaged the morphology of 
sporangiophores and sporangial shape in different lines with differential interference contrast optics and scanning electron microscopy (SEM). The results showed that PcFtsZ2 influenced both the frequency of branching in the sporangiophores and the shape of the sporangia. As shown in Figure 2A, Supplementary Fig. S8A, and Table 1, both silenced and overexpression lines produced more abundant branches on the sporangiophores, and three overexpression lines formed longer branches on the sporangiophores relative to the three silenced lines or controls $(P<0.01)$ (Fig. 2A; Supplementary Fig. S8A; Table 1). Additionally, the branches from the sporangiophores of the three silenced lines were gradually tapered to the apical initials, whereas the apical regions of branches from the sporangiophores of the three overexpression lines were swollen (Fig. 2A; Supplementary Fig. S8A).
As shown in Figure 2A and Supplementary Figure S8A, sporangia were significantly different in shape among all the different transformants and controls. All sporangia from the three silenced lines were malformed and lacked the distinct apical papillae. In contrast the morphology of sporangia from the three overexpression lines was nearly the same as those of the two controls. Therefore, we conclude that PcFtsZ2 is necessary for normal mycelial and sporangial development.

\section{Mycelial growth and sporangial development phenotypes of PcFtsZ2 silencing and overexpression lines are intensified at low temperatures.}

Fts $Z$ gene mutations in E. coli have been shown to cause an altered polar morphology at permissive temperatures and to block cell division at nonpermissive temperatures ( $\mathrm{Bi}$ and

\section{A}
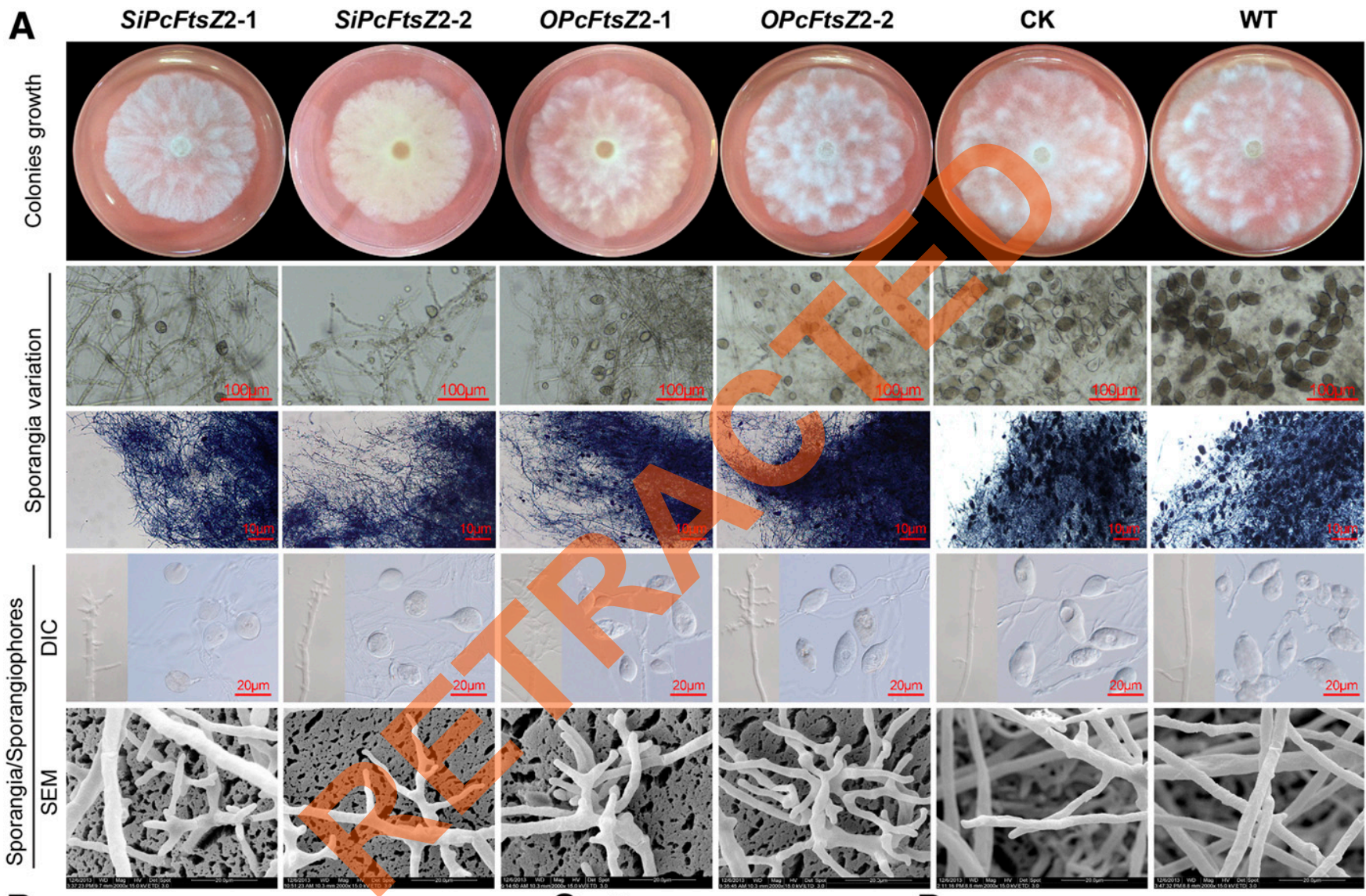

B

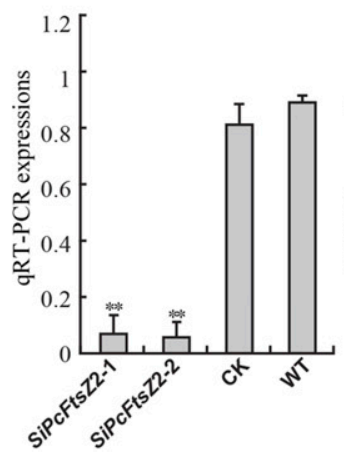

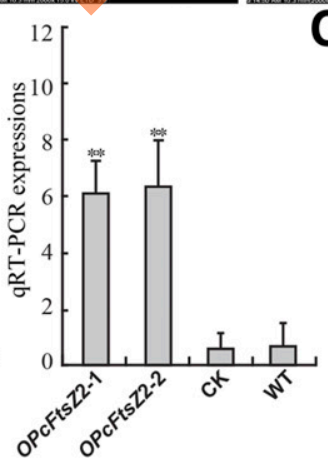

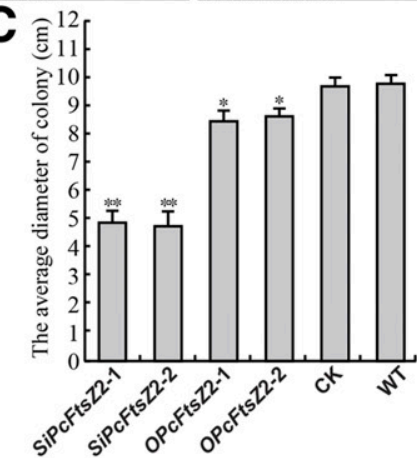

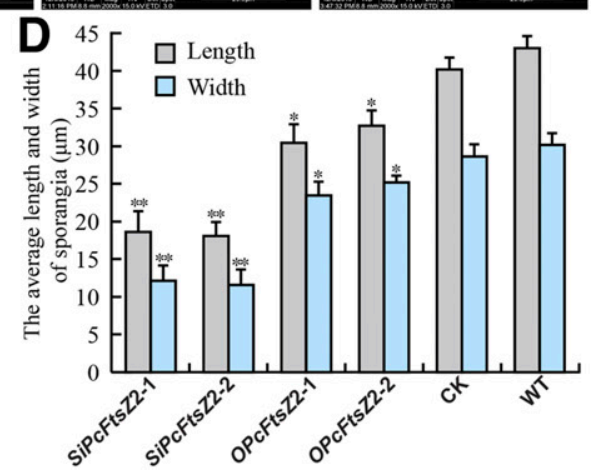

Fig. 2. Two silencing and two overexpression lines of PcFtsZ2 have pleiotropic effects on asexual development. A, The influence of PcFtsZ2 silencing or overexpression strains on growth of colonies (upper), number and sizes of sporangia (middle), and morphology of sporangia and sporangiophores (below). B, Transcript levels of PcFtsZ2 in two silencing and two overexpression strains were analyzed by quantitative reverse transcription-PCR. Three replicates for each sample were used to calculate standard errors. $\mathbf{C}$, Colony diameters of the different lines after culture for 7 days. $\mathbf{D}$, Variation of sporangia in the size ( $\mathrm{L}=$ length; $\mathrm{W}=$ width). The average size of sporangia was measured from at least 50 sporangia. Single asterisks $(*)$ indicate a significant difference from two controls $(P<0.05)$ by the Student's $t$ test and double asterisks $(* *)$ indicate an extremely significant difference from two controls $(P<0.01)$ by the Student's $t$ test. This assay was repeated at least three times. 
Lutkenhaus 1992). The ability to adapt to changing temperatures is an essential trait for foliar pathogens such as $P$. capsici. Thus, we measured the growth of colonies and the development of sporangia in different lines after 7 days at 15, 20, 25, 30, and $35^{\circ} \mathrm{C}$. Since the phenotypes of these silenced or overexpression lines have the same reaction to all the temperature ranges, two silenced lines (SiPcFtsZ2-1 and SiPcFtsZ2-2) and two overexpression lines (OPcFtsZ2-1 and OPcFtsZ2-2) were used for this analysis. $P$. capsici is a temperate pathogen and grows optimally at 25 to $30^{\circ} \mathrm{C}$ (Fig. 3A to E). Very poor growth and sporulation rates were observed for all lines at 15 and $35^{\circ} \mathrm{C}$. From 20 to $30^{\circ} \mathrm{C}$, the colony diameters of SiPcFtsZ2-1 and SiPcFtsZ2-2 were smaller than those of the two overexpression lines and the controls. The hyphal growth rates of OPcFtsZ2-1 and $O P c F t s Z 2-2$ were close to those of the two controls at $30^{\circ} \mathrm{C}$, but at 20 and $25^{\circ} \mathrm{C}$, their diameters were significantly smaller than those of the controls. At $20^{\circ} \mathrm{C}$, however, SiPcFtsZ2-1 and SiPcFtsZ2-2 did not form sporangia at all. At 25 and $30^{\circ} \mathrm{C}$, sporangial production of the silenced lines was about $40 \%$ of the controls, but the sporangia were significantly smaller than the controls $(P<0.05)$ and the sporangia of the silenced lines were malformed and lacked the normal apical papillae. At $20^{\circ} \mathrm{C}$, OPcFtsZ2-1 and OPcFtsZ2-2 formed fewer sporangia (50\% of controls, $P<0.01)$. These sporangia were significantly smaller $(P<0.01)$, malformed, and lacked the normal apical papillae. In contrast, at 25 and $30^{\circ} \mathrm{C}$, the shapes of the sporangia produced by OPcFtsZ2-1 and OPcFtsZ2-2 were similar to the two controls, although they were smaller than the two controls $(P<0.05)$ but larger than the two silenced lines $(P<0.05)$. Taken together, these results show that lower temperatures significantly intensify the phenotypes caused by silencing or overexpression of PcFtsZ2.

\section{PcFtsZ2 regulation of mitochondria and actin cytoskeleton.}

Since FtsZ proteins are involved in mitochondrial fission in other eukaryotes (Sheahan et al. 2005; Zottini et al. 2006) and PcFtsZ2 has a predicted mitochondrial targeting sequence, we used Mito-Tracker Green staining to visualize the number and arrangement of mitochondria in PcFtsZ2 silencing and overexpression lines. The mitochondria appeared to be distributed abnormally in both mycelia and sporangia of all six transformant lines, relative to WT and CK (Fig. 4A). Notably, in three silenced lines, there were fewer and bigger stained mitochondria in sporangia and mycelia. In the sporangia of overexpressed lines, the Mitotracker stain was brighter than in CK and WT (Fig. 4A). In mycelia of overexpressed lines, there were fewer large spots. The elevated levels of Mitotracker staining in the mycelia and sporangia of the three overexpression lines may be an indication of smaller and more numerous mitochondria.

To further investigate the possible structural basis of the mycelial and sporangial phenotypes caused by PcFtsZ2 misexpression, we observed the actin cytoskeleton in these lines using rhodamine-phalloidin staining and confocal microscopy (Fig. 4B). In the hyphae of WT and CK controls, actin plaques were arranged in a uniform belt-like helix pattern along the hyphae, as has previously been described (Bachewich and Heath 1998; Deora et al. 2008; Meijer et al. 2014). In sporangia of WT and CK, most of the staining was localized to Factin filaments and the plaques were oval-shaped. In both sporangia and hyphae of three silenced lines, plaques were more numerous than in WT and CK and were fewer than in the three overexpression lines. In the sporangia of three overexpressed lines, the plaques were more numerous, larger, and irregularly shaped relative to controls. In the hyphae of three overexpressed lines, the more numerous actin plaques were clustered together and were more closely positioned than in WT, CK, and three silenced lines, but a belt-like arrangement of plaques was still evident. In summary, silenced lines showed abnormal morphology and irregularities in the distribution of actin plaques and actin filaments in mycelia and especially in sporangia compared with either of the two controls. In contrast, in three overexpression lines, actin staining revealed an overabundance of intensely stained actin clumps or plaques. Thus, PcFtsZ2 protein expression directly

Table 1. Comparison of asexual growth of PcFtsZ2-silenced and overexpression lines relative to two controls ${ }^{\mathrm{a}}$

\begin{tabular}{|c|c|c|c|c|c|c|c|c|}
\hline \multirow[b]{2}{*}{ Phenotypes } & \multicolumn{3}{|c|}{ PcFtsZ2-silenced lines } & \multicolumn{3}{|c|}{ PcFtsZ2 overexpression lines } & \multicolumn{2}{|c|}{ Controls } \\
\hline & \multicolumn{2}{|c|}{$\begin{array}{ll}\text { SiPcFtsZ2-1 SiPcFtsZ2-2 } & \text { SiP }\end{array}$} & $\overline{\text { SiPcFtsZ2-3 }}$ & OPcFtsZ2-1 & OPcFtsZ2-2 & $\overline{\text { OPcFtsZ2-3 }}$ & CK & SD33 \\
\hline Colony diameter $(\mathrm{cm})^{\mathrm{b}}$ & $5.2 \pm 1.2^{* *}$ & $4.8 \pm 1.1 * *$ & $4.6 \pm 1.3 * *$ & $\pm 2.2^{*}$ & $8.2 \pm 2.1 *$ & $3.2 *$ & $9.6 \pm 1.4$ & $9.8 \pm 1.5$ \\
\hline Sporangia density ${ }^{c}$ & $32.5 \pm$ & $36.4 \pm 10.5^{* *}$ & $34.5 \pm 9.4^{* *}$ & $63.6 \pm 18.5^{*}$ & $67.3 \pm 14.5^{*}$ & 60.4 & $103.6 \pm 12.4$ & 110.4 \\
\hline Encystment $(\%)^{\mathrm{d}}$ & $83.0 \pm 10.5 * *$ & $81.3 \pm 14.1^{* *}$ & $76.5 \pm 12.4^{* *}$ & $52.4 \pm 12.6^{*}$ & $49.2 \pm 12.6^{*}$ & 46.4 & $34.7 \pm 9.6$ & 37.2 \\
\hline \multicolumn{9}{|l|}{ Germination (\%) } \\
\hline Cysts $^{\mathrm{e}}$ & $4 \pm 9.2 * *$ & $27.6 \pm 8.4 * *$ & $29.3 \pm 7.5^{* *}$ & $44.7 \pm 7.4 *$ & $47.7 \pm 8.2 *$ & $42.5 \pm 6.8^{*}$ & $86.7 \pm 7.3$ & $89.4 \pm 4.2$ \\
\hline Sporangia ${ }^{\mathrm{f}}$ & $17.6 \pm 4.2^{* *}$ & $19.3 \pm$ & $20.4 \pm 6$ & 35.7 & 38.5 & 32. & $79.7 \pm$ & 87.6 \\
\hline \multicolumn{9}{|l|}{ Germ tube length ${ }^{\mathrm{g}}$} \\
\hline Sporangia $(\mu \mathrm{m})$ & $2 * *$ & 47.8 & 44.5 & & 171. & 158 & 137.8 & 4.5 \\
\hline Cysts $(\mu \mathrm{m})$ & $* *$ & 20.3 & 17.5 & & & & & \\
\hline \multicolumn{9}{|l|}{$\begin{array}{l}\text { Branches on } \\
\text { sporangiophores }^{h}\end{array}$} \\
\hline Number & $11.6 \pm 2.7 * *$ & $10.7 \pm$ & $12.5 \pm 5.4^{* *}$ & $25.7 \pm 3.4 * *$ & $22.4 \pm 4.6^{* *}$ & $4 * *$ & $4.5 \pm 1.2$ & $5.0 \pm 1.3$ \\
\hline Length $(\mu \mathrm{m})$ & $9.4 \pm 3.6^{* *}$ & $8.8 \pm 2.8^{* *}$ & $7.9 \pm 3.4^{* *}$ & $21.8 \pm 6.7 * *$ & $19.6 \pm 7.3^{* *}$ & $24.4 \pm 6.4 * *$ & $5.4 \pm 1.5$ & 1.2 \\
\hline \multirow{2}{*}{\multicolumn{9}{|c|}{$\begin{array}{l}\text { a Silenced lines and overexpression lines derived from SD33. Independent sample } t \text { tests were used to compare each line to SD33. Single }(*) \text { and double } \\
\text { asterisks }(* *) \text { indicate } P<0.05 \text { and } P<0.01 \text {, respectively. Each test was duplicated three times. Values in the table are the mean } \pm \text { standard error. } \\
\text { b Based on } 7 \text { days of growth in } 10 \% \text { V8 juice agar medium at } 25^{\circ} \mathrm{C} \text {. }\end{array}$}} \\
\hline & & & & & & & & \\
\hline \multirow{3}{*}{\multicolumn{9}{|c|}{$\begin{array}{l}\text { c Number per square centimeter, based on counts of } 10 \text { plates made from } 7 \text {-day-old cultures grown in } 100 \text { mm plates at } 25^{\circ} \mathrm{C} \text { with } 80 \% \text { humidity. } \\
\text { d Percent of encysted zoospores in } 50 \mu \mathrm{l} \text { of zoospore suspension after } 1 \mathrm{~h} \text { of incubation, at } 25^{\circ} \mathrm{C} \text {, with } 80 \% \text { humidity. } \\
\text { e Percent of cysts forming germ tubes after } 2 \mathrm{~h} \text { of incubation, with vortexing to induce encystment, at } 25^{\circ} \mathrm{C} \text {, based on counting a minimum of } 100 \mathrm{cysts} \text { from } \\
\text { each strain. }\end{array}$}} \\
\hline & & & & & & & & \\
\hline & & & & & & & & \\
\hline \multicolumn{9}{|c|}{$\begin{array}{l}\text { f Percent of sporangia-formed germ tubes after } 6 \mathrm{~h} \text { of incubation, following vortexing to induce sporangia, at } 25^{\circ} \mathrm{C} \text {, based on counting a minimum of } 100 \\
\text { sporangia from each strain. }\end{array}$} \\
\hline \multicolumn{9}{|c|}{$\begin{array}{l}\mathrm{g} \text { The length of germ tubes formed from sporangia and cysts after } 6 \text { and } 2 \mathrm{~h} \text {, respectively, in } 10 \% \text { V8 liquid medium at } 25^{\circ} \mathrm{C} \text {, based on averaging a minimum of } \\
100 \text { germinated sporangia from each strain. }\end{array}$} \\
\hline & & & & & & & & \\
\hline
\end{tabular}


or indirectly affects the distribution of actin into filaments or plaques.

\section{PcFtsZ2 is essential for pathogenicity.}

To examine the function of PcFtsZ2 when the pepper was infected with $P$. capsici, the pepper leaves were inoculated with zoospores from three silenced lines (SiPcFtsZ2-1, SiPcFtsZ2-2, and SiPcFtsZ2-3), three overexpression lines (OPcFtsZ2-1, OPcFtsZ2-2, and OPcFtsZ2-3), WT, and CK strains. In contrast to the two controls, the lesions of the three silenced lines revealed striking differences (Fig. 5A and C; Supplementary Fig. S11A and C). In two controls, water-soaked lesions were evident at 1 day postinoculation (dpi) and, by 3 dpi, lesions covered large areas of the leaf ( 3.2 to $3.5 \mathrm{~cm}^{2}$ ). In contrast, three silenced lines caused only slight lesion development at $2 \mathrm{dpi}$, and, at $3 \mathrm{dpi}$, lesions diameters (approximately 0.7 to $0.9 \mathrm{~cm}^{2}$ ) were only about one third the size of WT or CK lesions $(P<$ 0.01) (Fig. 5A and C; Supplementary Fig. S11A and C). These results are consistent with our observations that silencing significantly reduces germination rates of sporangia, and the germ tubes formed by either cysts or sporangia are also shorter (Supplementary Fig. S12A and B; Table 1). The three overexpression lines produced visible lesions at $2 \mathrm{dpi}$, and lesions then became slightly larger (approximately 2.5 to $2.8 \mathrm{~cm}^{2}$ ) at 3 dpi (Fig. 5A, C; Supplementary Fig. S11A and C) but were significantly smaller than WT or CK lesions. These results are consistent with the lower germination rates of sporangia and cysts (Table 1) and the longer germ tubes formed by the sporangia and cysts of the OPcFtsZ2-1, OPcFtsZ2-2, and OPcFtsZ2-3 strains (Supplementary Fig. S12A and B; Table 1).

To further determine the reasons that cause the diminished virulence in these three silenced lines, a microscopic inspection of infection by germinating cysts on the leaves of a susceptible pepper was performed. The germinating cysts were examined at 6,12 , and $24 \mathrm{~h}$ after inoculation with zoospores. On the surface of the leaves, the germinating cysts of SiPcFtsZ2-1, SiPcFtsZ2-2, and SiPcFtsZ2-3 produced shorter germ tubes than did OPcFtsZ2-1, OPcFtsZ2-2, and OPcFtsZ2-3 or CK and WT (Fig. 5D, Supplementary Fig. S11D). Microscopic examination of the leaf surfaces showed that the epidermal cells of the leaf had not been penetrated by the germ tubes of SiPcFtsZ2-1, SiPcFtsZ2-2, and SiPcFtsZ2-3 after 6 or $12 \mathrm{~h}$ (Fig. 5D; Supplementary Fig. S11D). In contrast, germ tubes of OPcFtsZ2-1, OPcFtsZ2-2, and OPcFtsZ2-3 elongated much longer and started to penetrate the leaf tissues at 6 or $12 \mathrm{~h}$ (Fig. 5D, Supplementary Fig. S11D). At $24 \mathrm{~h}$, the germ tubes of the

A

B

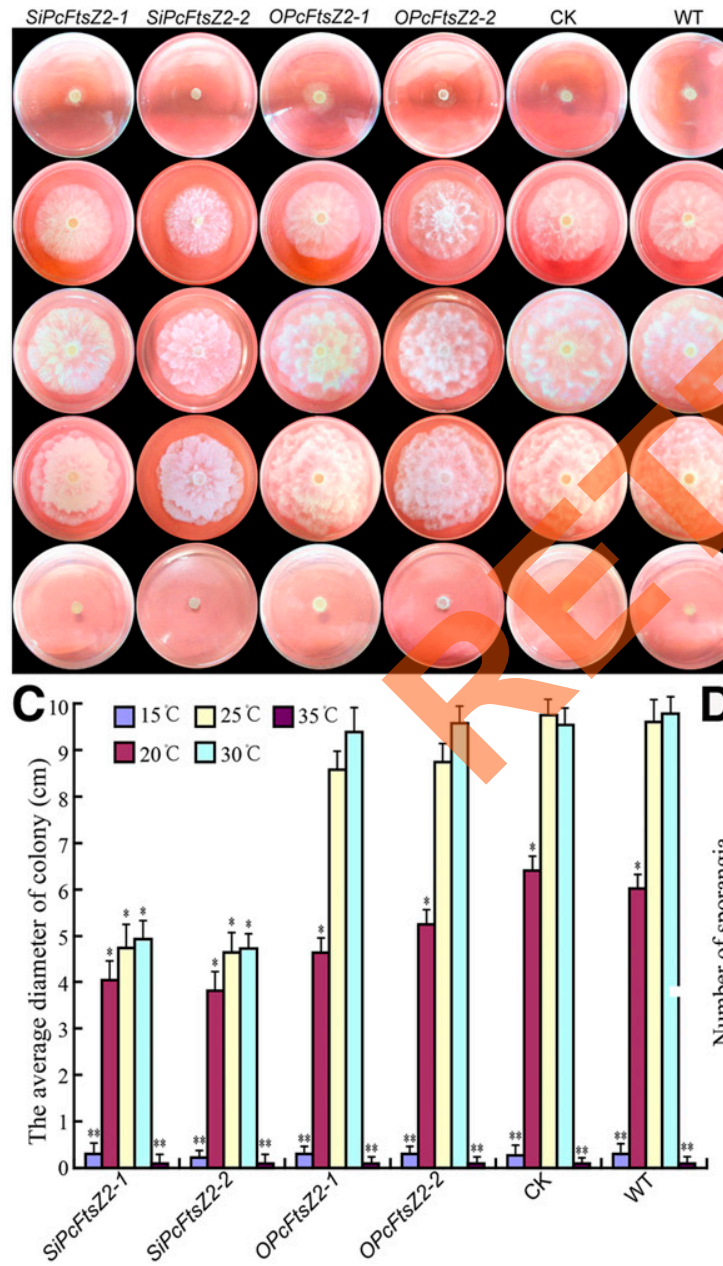

SiPcFtsZ2-1 SiPcFtsZ2-2
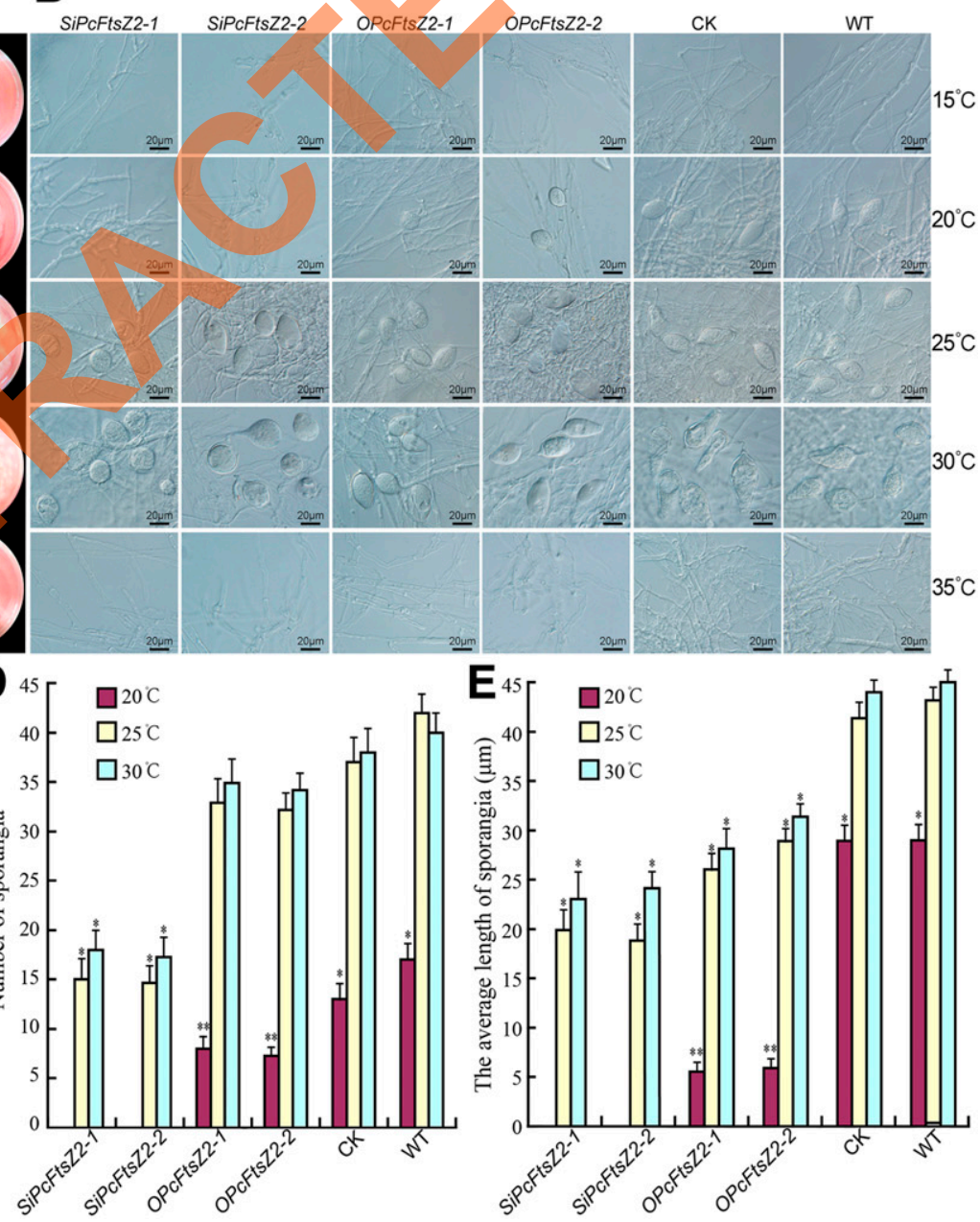

Fig. 3. Effect of temperature on mycelial growth and sporangial development in PcFtsZ2-silenced and overexpression lines. A, Hyphal growth of all the different lines on agar plates after 7 days. B, Effect of temperature on sporangial production by hyphae. Photos were taken at 9 days. $\mathbf{C}$, The average diameter of colonies by different lines after incubation for 7 days. Growth rates of colonies were measured from at least 25 plates. D, Numbers of sporangia produced at different temperatures by different lines. E, Variation length of the sporangia. The average length of sporangia was measured for at least 50 sporangia. Single asterisks $(*)$ indicate a significant difference $(P<0.05)$ by Student's $t$ test and double asterisks $(* *)$ indicate an extremely significant difference $(P<0.01)$ by Student's $t$ test. This assay was repeated at least three times. 
silenced lines were longer and started to penetrate the tissue. In both respects, however, disease progression in these three lines lagged behind the controls at 12 or $24 \mathrm{~h}$ (Fig. 5D; Supplementary Fig. S11D). Additionally, more extensive development of invasive mycelium was observed in WT and CK than in
OPcFtsZ2-1, OPcFtsZ2-2, and OPcFtsZ2-3 at $24 \mathrm{~h}$ (Fig. 5B, Supplementary Fig. S11B), consistent with the efficient germination and germ tube growth of the cysts (Fig. 5D; Supplementary Fig. S11D). Furthermore, there were less hyphae distributed on the surface of the leaves after inoculation with
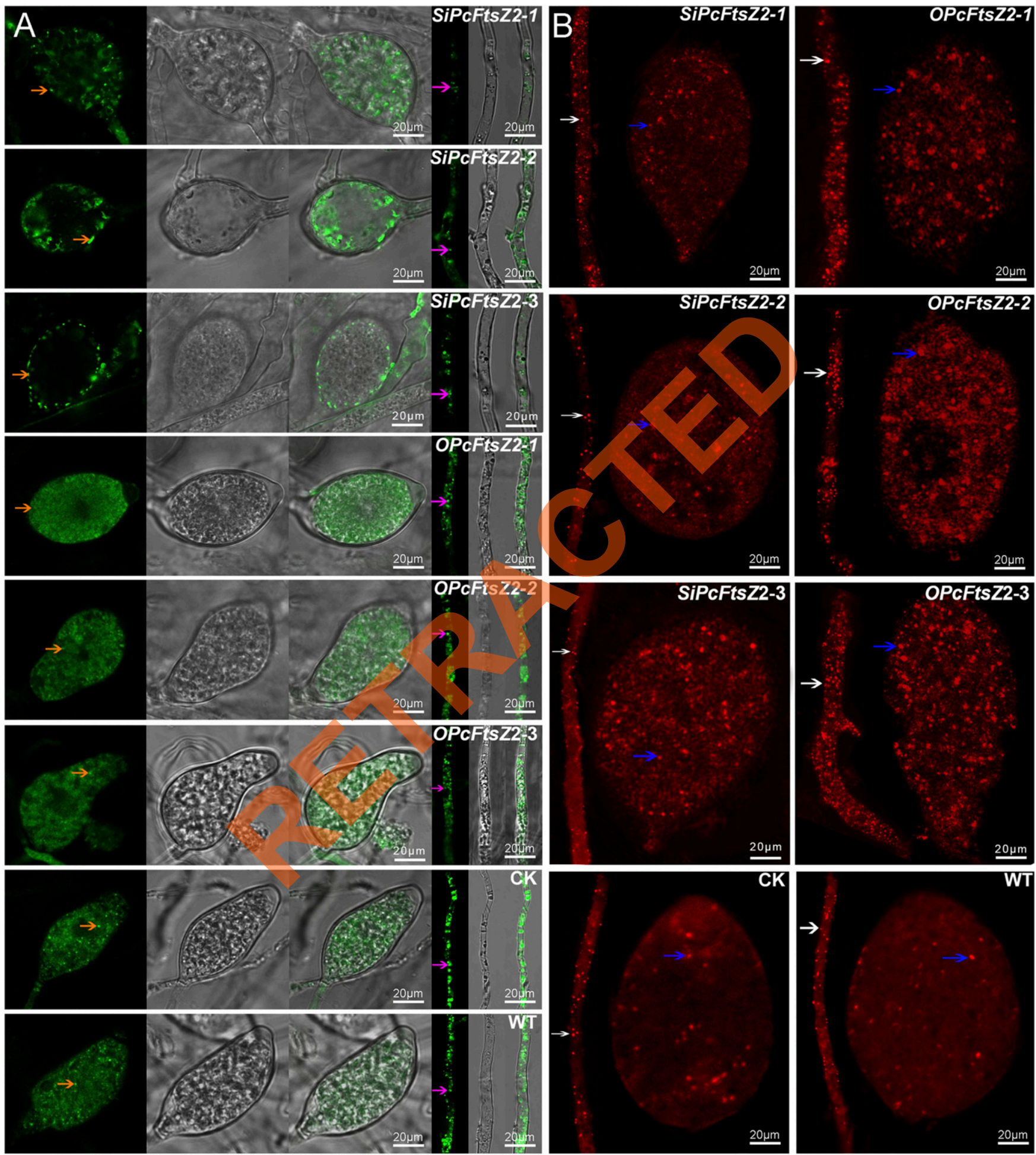

Fig. 4. PcFtsZ2 regulation of mitochondria and actin cytoskeleton. A, Confocal micrographs of Mito-Tracker Green-stained mitochondria in sporangia and mycelia of PcFtsZ2-silenced and overexpressed strains contrast to two controls. Orange arrows point to mitochondria in sporangia of the three silencing and three overexpression lines compared with the empty vector control (CK) and wild type (WT). Magenta arrows point to mitochondria in mycelia of the three silencing and three overexpression lines compared with CK and WT. B, Confocal micrographs of rhodamine phalloidin-stained actin cytoskeleton in sporangia and mycelia of PcFtsZ2 mis-expression lines. Images are false-colored red. White arrows point to actin plaques in hyphae of the three silencing and three overexpression lines compared with CK and WT. Blue arrows point to actin plaques in sporangia of the three silencing and three overexpression lines compared with CK and WT. Each assay was repeated at least three times. An empty vector transformant was used as CK; SD33 was used as WT. 
A
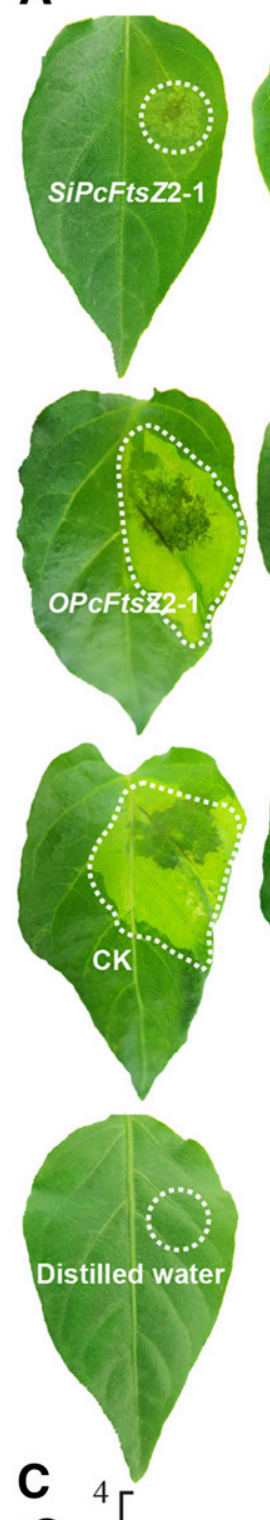

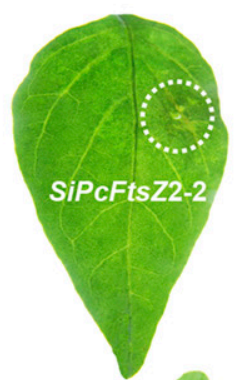

B
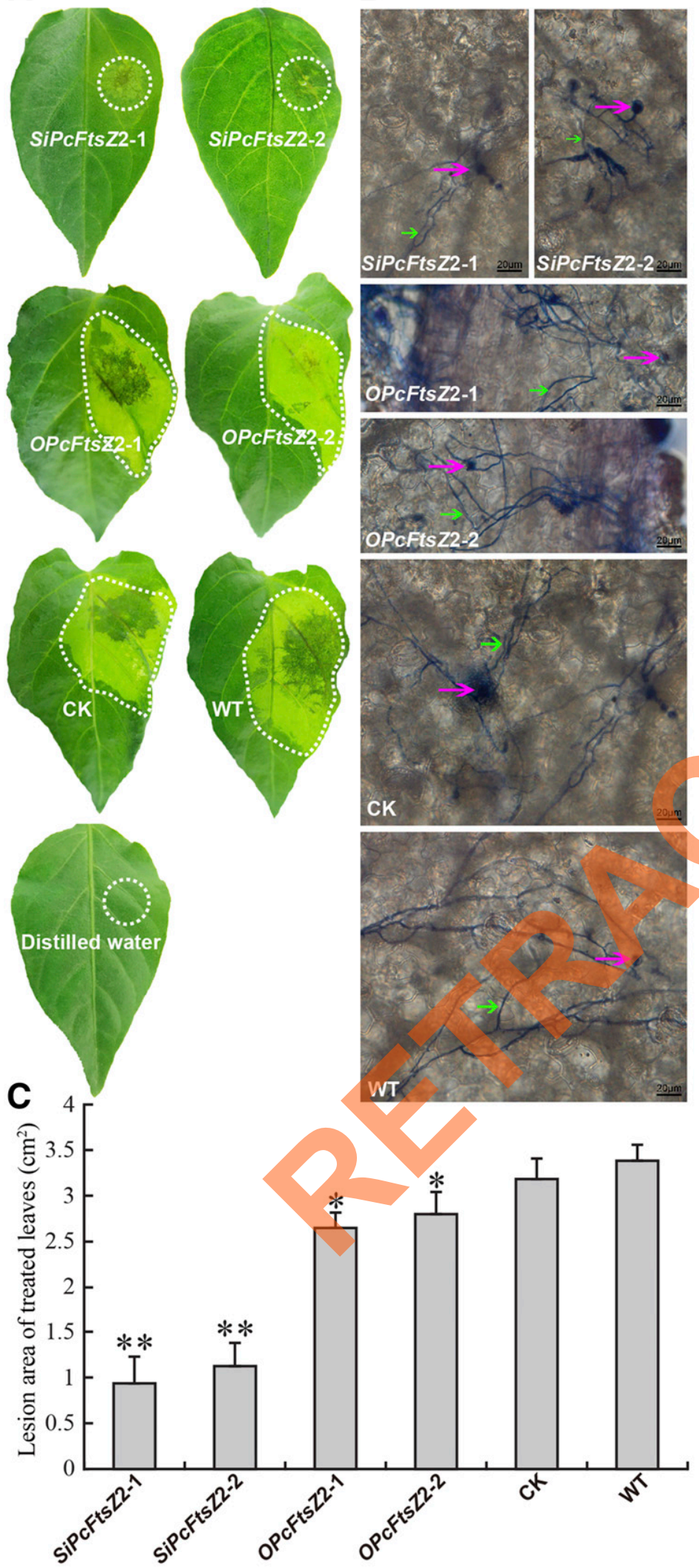

D

$6 h$

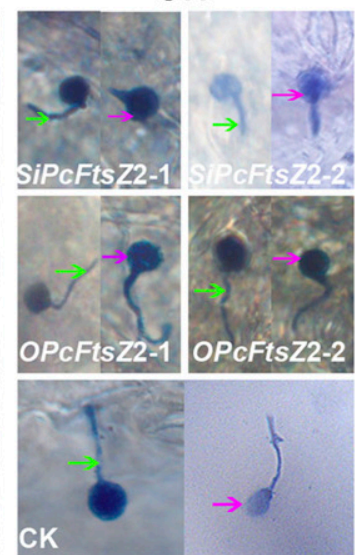

CK
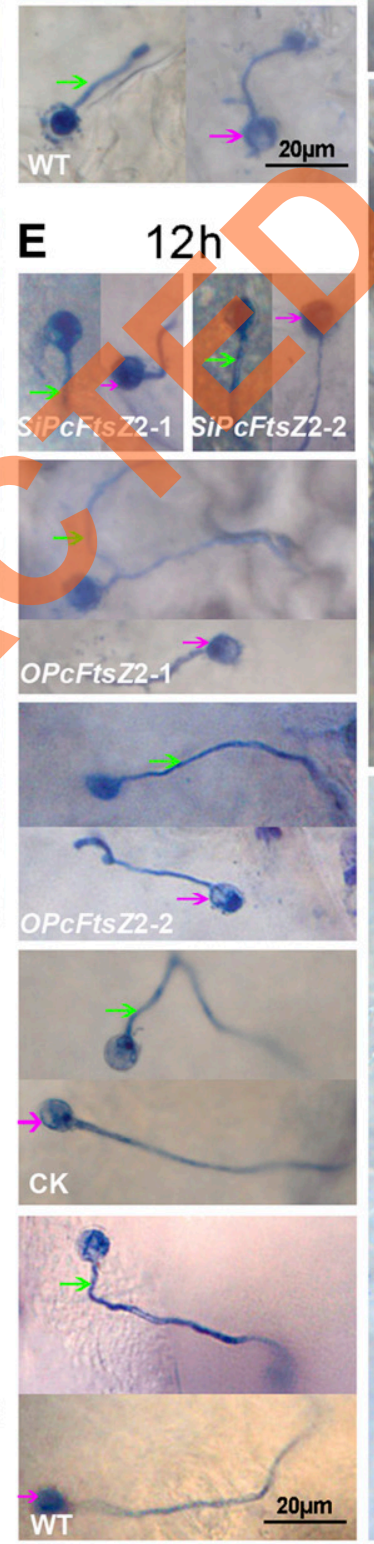

\section{F $24 \mathrm{~h}$}
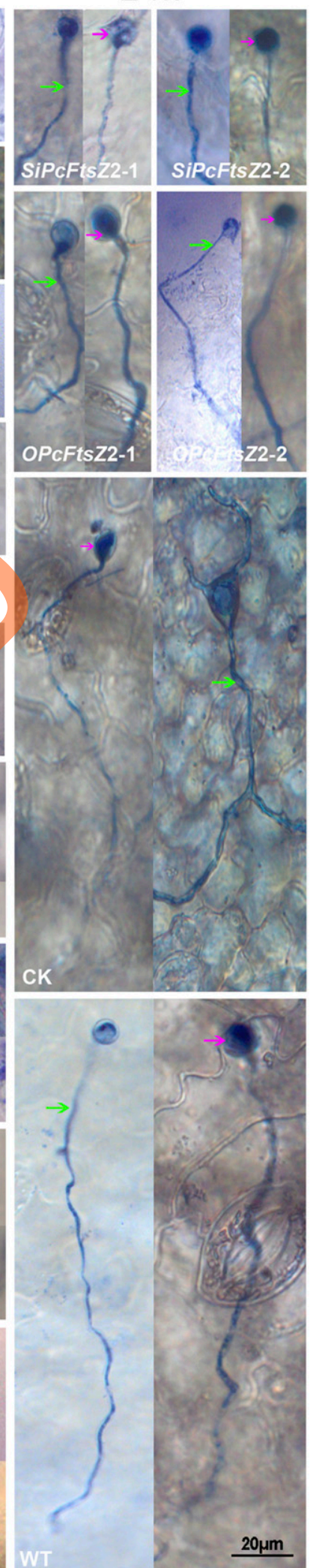

Fig. 5. Silencing and overexpression of PcFtsZ2 alters virulence. A, Pepper leaflets were inoculated with zoospores of PcFtsZ2 mis-expression strains and two control strains. Photographs were captured at 3 days postinoculation (dpi). B, Hyphal distribution in the tissues of pepper leaves after inoculation with zoospores of these different strains at $24 \mathrm{~h}$ postinoculation. Magenta arrows point to germinating cysts. Green arrows point to germ tubes formed by cysts as they penetrated into the epidermal cells of the leaf. C, Lesion areas were measured at 3 dpi. Bars represent the mean \pm standard error from 14 leaves. Single asterisks (*) indicate a significant difference from wild type (WT) $(P<0.05)$ by Student's $t$ test and double asterisks $(* *)$ indicate an extremely significant difference from WT $(P<0.01)$ by Student's $t$ test. $\mathbf{D}, \mathbf{E}$, and $\mathbf{F}$, Microscopic observation of cyst germination and germ tube penetration on the surface of pepper leaves at 6, 12, and $24 \mathrm{~h}$, respectively; typical structures are shown. Magenta arrows point to germinating cysts. Green arrows point to germ tubes formed by cysts. Each experiment was repeated at least three times. 
the silenced strains (Fig. 5B; Supplementary Fig. S11B). Taken together, altered expression of PcFtsZ2 affects sporangia and cyst germination rates, germ tube lengths, hyphae growth, and the ability of hyphae to penetrate pepper tissues. It is hardly surprising that these changes significantly impair the pathogenicity of transformants.

\section{DISCUSSION}

The coordination of hypha polarity and cell architecture is a fundamental requirement for normal growth. Mitochondria generate the energy that enables cell growth and division. The FtsZ protein, a key effector of cellular fission in bacteria $(\mathrm{Bi}$ and Lutkenhaus 1991; Ben-Yehuda and Losick 2002; Addinall et al. 1997), has also been identified as an important player in the fission of mitochondria and chloroplasts in a subset of eukaryotes (Kiessling et al. 2000). Homologs of FtsZ have previously been identified bioinformatically in oomycetes, along with homologs of FtsZ-associated cell division regulators MinC, MinD, and MinE (Leger et al. 2015). However, no functional analysis of the oomycete FtsZ proteins has been performed. Here, we demonstrate that regulation of PcFtsZ2 expression levels is a requirement for normal asexual growth, development, and virulence of $P$. capsici. First, we surveyed sequences of oomycete genomes to demonstrate that all oomycetes, including $P$. capsici, have two divergent copies of FtsZ and that these proteins are highly conserved. The PcFtsZ1 and PcFtsZ2 genes encode proteins having around 50\% amino acid identity with the conserved 318-amino acid core region of E. coli $\mathrm{FtsZ}$ and around $60 \%$ identity with $\alpha$-proteobacterial sequences. In PcFtsZ1 and PcFtsZ2 proteins, the more divergent sequences outside the core consist of the mitochondrial transit peptide sequences, in the case of the N-termini, and the variable spacer region at the $\mathrm{C}$-termini, similar to the bacterial FtsZ proteins. Furthermore, both genes were annotated in $P$. capsici genome sequence as "FtsZ" by several hidden Markov models, including TIGRFAM TIGR00065. Thus, both genes are likely correctly identified as co-orthologs of the E. coli FtsZ gene. As shown in Supplementary Figure S4, PcFtsZ1, PcFtsZ2, and their oomycete homologs are strongly conserved with $E$. coli and $\alpha$-proteobacterium FtsZ sequences. These two proteins contain three conserved motifs found in a wide range of prokaryotic organisms (Ma and Margolin 1999). The short 16-amino acid region at the extreme C termini of the bacterial protein, identified as the requirement for interactions (Hale et al. 2000), was not present in either sequence. The Phytophthora FtsZ2 sequences carry a variable C-terminal domain and, at their extreme $\mathrm{C}$ termini, there is a nine-residue sequence that is well conserved across oomycete genomes. This sequence shares only two conserved residues with the bacterial C-terminal sequence. Both PcFtsZ1 and PcFtsZ2 are singlecopy genes, as anticipated for highly conserved housekeeping genes in Phytophthora spp.

To confirm whether PcFtsZ1 and PcFtsZ2 are functional homologs of $E$. coli FtsZ, we carried out heterologous expression of PcFtsZ1 and PcFtsZ2 in E. coli JM109. Overexpression of PcFtsZ1, PcFtsZ2, and EcFtsZ alone or as GFP fusions, resulted in a filamentation phenotype (Fig. 1B and D; Supplementary Fig. S7A and B). The GFP fusions of three proteins accumulated in a highly regularly punctate pattern within filamentous cells. Ma et al. (1996) speculated that the excess FtsZ protein was accumulating at the potential sites of cell division but could not complete the division process due to lack of partner proteins, such as FtsZA. Our observations showed that PcFtsZ1 and PcFtsZ2 could trigger the same phenotypes as EcFts $Z$ and support the bioinformatic prediction that PcFtsZ1 and PcFtsZ2 are functional homologs of bacterial FtsZ genes.
Using silencing and overexpression of PcFtsZ2 in P. capsici, we investigated its various roles in mycelial growth, sporangial development and direct germination, cyst germination, and infection of pepper leaves. Here, silencing of PcFtsZ2 led to colony expansion in vitro that was more restricted but also much denser than two controls (Fig. 2A; Supplementary Fig. S8A; Supplementary Fig. S10A). Silencing of PcFtsZ2 almost completely suppressed the production of aerial hyphae (Supplementary Fig. S10A). Overexpression led to a greater rate of colony expansion, with abundant aerial hyphae but a sparse mycelial mass relative to the controls (Fig. 2A; Supplementary Figs. S8A and S10B). Close inspection of the sporangiophores revealed that many branches were mishappen in both the silenced and overexpressed lines (Fig. 2A; Supplementary Fig. S8A). Branching of sporangiophores in the overexpression lines were more extensive compared with silenced lines. In addition, the sporangia from the silenced lines were the smallest in size and the fewest in number (Fig. 2A; Supplementary Fig. S8A; Table 1). As shown in Figure 2A and Supplementary Figure S8A, these sporangia were malformed and lacked distinct apical papillae, in contrast to those of the overexpression lines and WT and CK. Moreover, sporangia produced by the overexpression lines OPcFtsZ-1, OPcFtsZ-2, and OPcFtsZ-3 were similar in shape to the controls. Thus, PcFtsZ2 is critical for regulating the sporangiophore and sporangial development in $P$. capsici.

Mitochondrial staining was substantially reduced in the silenced lines, suggesting a reduced cellular volume of functioning mitochondria (Fig. 4A). In the sporangia of silenced lines, much of the staining was associated with larger dots, an indication that mitochondria were larger than in the sporangia of other cell lines. In contrast, the sporangia of overexpressed lines contained fewer intense spots and mitochondria were more uniform and denser than the two controls. This pattern of staining conformed to what might be expected if mitochondria were smaller and evenly distributed. Taken together, these results suggested that expression levels of FtsZ2 affected the number of mitochondrial divisions. Silencing reduced the number of actin plaques, while overexpression increased both the number and size of actin plaques (Fig. 4B). In summary, PcFtsZ2 mis-expression contributed to changes in morphology and numbers of mitochondria and, also, resulted in alternating the number and size of actin plaques in the cell of $P$. capsici. Therefore, our results confirm that asexual characteristics of $P$. capsici sporangia are closely connected with the number, size, and the distribution of both mitochondria and actin.

There are two FtsZ proteins in $P$. capsici, based on the characterization of the two orthologous FtsZ proteins from members of genus Dictyostelium; thus, they have different roles in mitochondrial fission. Decreased levels of PcFtsZ2 might be expected to reduce fission rates but increased levels of one of the PcFts $Z$ genes might not be sufficient to enhance fission rates. The potential homologs of MinC, MinD, and MinE have been identified in oomycetes (Leger et al. 2015). In bacteria, these proteins direct the positioning of the $\mathrm{Z}$ ring, which determines where the septum will form. Other proteins that span the mitochondrial membranes to connect FtsZ proteins with dynamins, such as Drp1, have not been identified, since there are no orthologs of other bacterial proteins involved in bacterial fission in stramenopiles. However, it is possible that an excess expression of PcFtsZs may disrupt the interaction of PcFtsZs with partner proteins that also span the mitochondrial membranes to connect the organelles to actin filaments (Moore and Holzbaur 2018).

Several mechanisms could account for the developmental phenotypes seen in both PcFtsZ2-silenced and overexpressed lines. Altering the ratio of the two FtsZ proteins could 
negatively impact energy production by the mitochondria (Araki et al. 2003; Stokes and Osteryoung 2003). Such changes could also negatively impact cytoskeleton dynamics. Notably, at suboptimal temperatures, the developmental phenotypes of both silenced and overexpressed lines were more severe (Fig. $3)$. Energy limitations could potentially account for the increased severity of phenotypes at suboptimal temperatures (Bi and Lutkenhaus 1992). In the chloroplast, FtsZ proteins interact with integral membrane protein partners, connecting FtsZ proteins to the actin cytoskeleton (Chen et al. 2018). Changes in the dynamics of fusion and fission rates alter mitochondrial shapes and, thus, indirectly affect their movement along the cytoskeleton of the cell (Moore and Holzbaur 2018). In humans, defects in mitochondrial dynamics are associated with a number of disease states (Westermann 2010). Therefore, the results described here are consistent with these findings that careful regulation of mitochondrial fission is a requirement for normal growth in eukaryotic cells.

The aberrant growth and development of hyphae and sporangia caused by PcFtsZ2 mis-expression also performed a strong effect on indirect germination of sporangia and cysts. Germ tubes produced by either sporangia or cysts were the shortest in silenced lines (Fig. 5D; Supplementary Figs. S11D and S12; Table1), while. in overexpression lines, germinating sporangia produced the longest germ tubes (Supplementary Fig. S12) and germinating cysts produced significant long germ tubes (Fig. 5D; Supplementary Fig. S11D). To summarize, misexpression of PcFtsZ2 caused disruption of the normal asexual growth and development of $P$. capsici. Therefore, we conclude that specific regulation of the levels of PcFtsZ2 is required for normal asexual characteristics of $P$. capsici.

Oomycete infection to host tissues is an energy-intensive process, and hyphal tips must overcome physical barriers and both constitutive and induced chemical defenses. Thus, in either oomycetes or filamentous fungi, modulation of the actin cytoskeleton at the tip of invasive hyphae has been shown to be a mechanism to reinforce the level of intrusive force (Suei and Garrill 2008). Thus, the disorganization of the actin cytoskeleton displayed in PcFtsZ2 silenced or overexpressed lines could also potentially impair virulence. We observed that silenced lines of PcFtsZ2 were severely reduced in virulence. Three silenced lines, strains SiPcFtsZ2-1, SiPcFtsZ2-2 and SiPcFtsZ2-3, caused the pepper leaves to produce abnormally small lesions and a strongly sparse or reduced distribution of hyphae in lesion tissues (Fig. 5A, B, and C; Supplementary Fig. S11A, B, and C). The silenced lines also generated very short germ tubes in cysts in the lesion tissues (Fig. 5D, E, and F; Supplementary Fig. S11D, E, and F). The overexpression lines also exhibited slightly reduced lesion sizes (Fig. 5A and C; Supplementary Fig. S11A and C). Here however, the reduction in lesion size might also be due to more frequent branching of hyphae that might affect the rate of penetration into new tissues, leading to reduced ability of the pathogen to cause disease.

Cytoskeletal proteins are unlikely to function independently of each other (Moore and Holzbaur 2018) and we note that PcFtsZ2 resulted in changes in the branching patterns of hyphae and the distribution of the actin cytoskeleton. In overexpression lines, both hyphal growth and branching rates were significantly stronger than those of two controls and also showed a huge increase of both the number and the size of actin plaques. This combination of rapid hyphal extension along with the redistribution of more actin into actin plaques seemed incompatible with the model of oomycete hyphal growth that was described using only Lifeact-eGFP (Meijer et al. 2014). In overexpressed lines, we noted a higher rate of hyphal elongation, but actin staining showed a greater number of larger irregular plaques. In contrast, florescence movies of hyphae expressing
Lifeact-eGFP showed that actin plaques were relatively immobile and could form and dissociate very quickly (approximately 10 to $15 \mathrm{~s}$ ) but had very long turnover times (average $78 \mathrm{~min}$ ) relative to those reported for fungi $(15 \mathrm{~s})$. Actin filaments are both dynamic and interconnected to other cytoskeletal proteins such as microtubules (Bachewich and Heath 1998). Filamentous actin mediates mitochondrial fission (Moore and Holzbaur 2018) and alterations in the expression of FtsZ2 may affect assembly of protein complexes that direct mitochondrial fission. Transformation of cell lines that are altered in the expression of PcFtsZ2 together with Lifeact-eGFP could add to our understanding of how protein-protein interactions with PcFtsZ2 affect mitochondrial positioning and fission rates and, in turn, control hyphal growth and branching rates. The genomes of oomycetes contain many examples of genes, such as FtsZ, that distinguish them from fungi (Judelson 2017). Functional analysis of more evolutionarily conserved proteins may be useful in identifying new management strategies for oomycete pathogens.

\section{MATERIALS AND METHODS}

Cultivation of the $\boldsymbol{P}$. capsici strain and pepper seedlings.

Experimental isolates were maintained on $10 \%$ V8 agar (Erwin and Ribeiro, 1996), and this media was also used in growth assays. The strongly virulent $P$. capsici isolate SD33 was isolated in our laboratory $\mathrm{Fu}$ et al. (2015). The mycelia, sporangia, zoospores, cysts, and germinated cysts were obtained as described by Hua et al. (2013) with minor adjustments. We manually counted the number of sporangia and zoospores using previously described methods (Fu et al. 2015). Each experiment was replicated at least three times. A susceptible pepper cultivar (Capsicum annuum inbred line 06221) was selected for estimating the virulence of the tested strains. The pepper seedlings were cultivated and grown with reference to methods reported by $\mathrm{Fu}$ et al. (2015).

\section{PcFtsZ2 gene cloning and sequence analysis.}

The Fts $Z$ genes were identified in the genome sequence of $P$. capsici using the full-length amino acid sequences of FtsZ from Escherichia coli. PsFtsZ (P. sojae) and PrFtsZ ( $P$. ramorum) sequences were downloaded from the Department of Energy Joint Genome Institute (JGI) website, and PiFtsZ (P. infestans) and PpFtsZ (P. parasitica) sequences were downloaded from the Broad Institute website (Supplementary Table S2). Other sequences of FtsZs were obtained using National Center for Biotechnology Information BLAST searches. The accession numbers of FtsZ sequences used in the analyses are given in Supplementary Table S2. Multiple alignment of FtsZ sequences was done using Clustal X 2.0 (Thompson et al. 1997). Phylogenetic trees were generated by neighbor-joining using MEGA5 beta with the default parameters. Nodal support of the trees was estimated by bootstrapping, with 1,000 pseudoreplicate data sets. The conserved functional domains of all those amino acid sequences were predicted using the online software SMART and InterProScan.

To amplify PcFtsZ1 and PcFtsZ2 from P. capsici SD33, primers were designed based on the sequences of PcFts $Z 1$ and PcFtsZ2 in the JGI database (Supplementary Table S1). Genomic DNA was extracted from the $P$. capsici strain as previously reported (Tyler et al. 1995). PCR amplification and the sequence analysis were done as previously reported ( $\mathrm{Fu}$ et al. 2015) with minor adjustments.

\section{SYBR green real-time RT-PCR analysis.}

To evaluate the transcript levels of PcFtsZ2 in different asexual stages (mycelium, sporangia, zoospore, cyst, and germinating cyst) and during the early phase of infection of the 
susceptible pepper cultivar inbred line 06221, samples were collected at $1.5,3,6,12,24,48$, and $72 \mathrm{~h}$ postinoculation (hpi) as previously described ( $\mathrm{Fu}$ et al. 2015). All samples were immediately put into liquid nitrogen and were stored at $-80^{\circ} \mathrm{C}$ for RNA extraction. Total RNA was extracted from all the samples using the NucleoSpin RNA II kit (Invitrogen), following manufacturer protocols. Primers were designed, to anneal specifically to PcFtsZ2, using Primer express software version 3.0 for SYBR green real-time PCR (Supplementary Table S1). PcFtsZ2 expression in the different development and infection stages was normalized using the constitutively expressed genes $\beta$-actin (number 189084454), $\beta$-tubulin (number 50660663) and $U b c$ (number 23394351) of $P$. capsici and $\beta$-actin (number 45861744) of pepper (Yan and Liou, 2006). PcFtsZ2 transcript levels of all above samples were determined using the ICycler IQ RT-PCR and SYBR primer Script RT-PCR kit, following the manufacturer protocols. PCR reaction conditions were similar to those previously described, with minor adjustments (Fu et al. 2015). The values of the threshold cycles (CT) were calculated automatically by the instrument, and the fold changes of PcFtsZ2 were estimated utilizing the equation $2^{-\Delta \Delta \mathrm{CT}}$ as previously described by Pfaffl (2001). Each sample timepoint included at least three replicates.

\section{Heterologous expression \\ of PcFtsZ1, PcFtsZ2, and EcFtsZ in E. coli.}

E. coli JM109 was collected from Promega. E. coli JM109 growth and culture conditions were as previously reported (Yee et al. 2012). The DNA of E. coli JM109 was extracted using a genomic DNA isolation kit (Bacteria Genomic DNA kit) following the manufacturer protocols. The full-length of EcFtsZ was amplified from E. coli JM109 using primers JM109EcFtsZ-F and JM109-EcFtsZ-R (Supplementary Table S1), which contain, at their ends, the BamHI and HindIII restriction sites, respectively. The PCR reactions were carried out as previously described (Sambrook and Russell 2001)

PcFtsZ1, PcFtsZ2, and EcFtsZ were individually inserted into pET28a vector (containing His tag) to generate pET28a-PcFtsZ1, pET28a-PcFtsZ2, and pET28a-EcFtsZ, as described previously (Maier et al. 2015). These constructs were then transformed into E. coli JM109, respectively. pET28a was used as CK. E. coli JM109 was used as WT. The cloning junctions were confirmed by DNA sequencing. pET28a-PcFtsZ1, pET28a-PcFtsZ2, pET28aEcFtsZ, and pET28a were individually expressed in JM109 and were monitored by SDS-PAGE using Coomassie blue staining (Liu and Naismith 2009). Anti-His antibody was used to detect the PcFtsZ1, PcFtsZ2, and EcFtsZ proteins by Western blot, and protein loading was indicated by Ponceau staining. The PcFtsZ1, PcFtsZ2, and EcFtsZ protein separation and blot membrane preparation were carried out essentially as described by Wang et al. (2013). Each assay was repeated at least three times. Morphology of all experimental strains was observed by microscopic examination and representative photographs were taken using an OLYMPUS BX53 microscope. Photographs were subsequently processed using the Autolevel and Autocontrast features of Adobe Photoshop 9.0.

\section{Heterologous expression of PcFtsZ1-GFP, PcFtsZ2-GFP, and EcFtsZ-GFP in $E$. coli.}

The pEHIS-GFP-TEV vector was provided by the Center for Biomolecular Science. To create pEHIS-GFP-TEV-PcFtsZ1, pEHIS-GFP-TEV-PcFtsZ2, and pEHIS-GFP-TEV-EcFtsZ, all PCR-purified products of PcFtsZ1, PcFtsZ2, and EcFtsZ were individually digested with restriction enzymes and were then ligated into pEHIS-GFP-TEV vector (Yang et al. 2011). PcFtsZ1 and PcFtsZ2 were PCR amplified for constructing into
pEHIS-GFP-TEV, using primers PcFtsZ1-EcoRI-F and PcFtsZ1-HindIII-R for PcFtsZ1 and PcFtsZ2-EcoRI-F and PcFtsZ2-HindIII-R for PcFtsZ2 (Supplementary Table S1). PCR products were confirmed by sequencing. pEHIS-GFPTEV-PcFtsZ1, pEHIS-GFP-TEV-PcFtsZ2, pEHIS-GFP-TEVEcFtsZ, and pEHIS-GFP-TEV were then individually transformed into JM109. CK (pEHIS-GFP-TEV) and WT (JM109) were used as controls. The cloning junctions were confirmed by DNA sequencing. The transcript levels of PcFtsZ1, PcFtsZ2, and EcFts $Z$ in these transformants were measured by qRT-PCR as described above. The transcript levels of EcFtsZ in E. coli cells were used to compare the expression levels of PcFtsZ1, PcFtsZ2, and EcFtsZ in the transformants. The pEHIS-GFPTEV-PcFtsZ1, pEHIS-GFP-TEV-PcFtsZ2, pEHIS-GFP-TEVEcFtsZ, pEHIS-GFP-TEV were then individually expressed in E. coli JM109, and cellular extracts were analyzed by SDSPAGE using Coomassie blue staining. PcFtsZ1, PcFtsZ2, and EcFtsZ proteins were individually detected by Western blot using GFP-antibody (1:2,000; Abmart), and equal protein loading of gel lanes was verified by Ponceau staining. Each experiment was repeated at least three times. Morphology of the GFP-fusion transformants was imaged with an Olympus BX-53 microscope. Images for green fluorescence, excitation and emission settings were 460 and 480 to $540 \mathrm{~nm}$ for GFP. Photographs were subsequently processed using the Autolevel and Autocontrast features of Adobe Photoshop 9.0.

\section{Plasmids constructs for transformation of $\boldsymbol{P}$. capsici.}

The plasmids pHam34 and pHspNpt were used for transformation. Primers for constructing transformation are listed in Supplementary Table S1. To generate the constructs, DNA fragments were isolated from SD33 DNA and were digested with SmaI, which were then cloned into pHam34. The insertion sequences were confirmed by DNA sequencing. The production of $P$. capsici protoplasts and the transformation procedures were performed as previously described with minor adjustments (McLeod et al. 2008). PcFtsZ2-silenced and overexpressed lines were produced using well-established protocols (Ah-Fong et al. 2008; Fu et al. 2015; McLeod et al. 2008). The transcript levels of PcFtsZ2 in the strains of transformants were determined using qRT-PCR and were analyzed as described above. Each experiment was repeated at least three times.

\section{Assessment of colony growth, sporangiophore, sporangial morphology, and germination of the transformations.}

All strains were subcultured twice on G418-free 10\% V8 juice agar medium for growth assays. Sterile $10 \%$ V8 juice $(20 \mathrm{ml})$ in Petri dishes was used to inoculate all the strains for observing the morphology of sporangia, sporangiophores, or hyphae. The sporangia and mycelia at 7 days were collected and were stained with trypan blue (Zhu et al. 2016). Colony diameters was measured at $1,3,5$, and 7 days over temperature ranges of $15,20,25,30$, and $35^{\circ} \mathrm{C}$, respectively. For assessment of sprorangial production, 3-day-old hyphae were rinsed with water three times and were then incubated for $1 \mathrm{~h}$ at $4^{\circ} \mathrm{C}$. After 9 days of incubation on agar plates, hyphae and sporangia were stained with aniline blue for microscopic examination (Fabro et al. 2011). The sporangia sizes and zoospore number were determined as previously reported (Zhu et al. 2016). The germination of sporangia was measured by previous study $(\mathrm{Fu}$ et al. 2015). The longest hyphal tips were measured in all cases in which multiple germ tubes were produced.

\section{Light microscopy and SEM.}

We used an Olympus BX-53 microscope to observe the mature sporangia, sporangiophores, or hyphae of the different transformation lines, CK, and WT (SD33) strains. All images 
of these samples were processed using Adobe Photoshop (Fu et al. 2015). A scanning electron microscope was used to observe the morphology of hyphae or sporangiophores in the different strains. The samples of sporangiophores or hyphae were prepared according to previous descriptions (Fu et al. 2015). Images were obtained using a Phillips XL30 FEG microscope. Each experiment was conducted at least three times.

\section{Actin, mitochondria staining, and} confocal laser scanning microscopy.

Sporangia, sporangiophores, and hyphae of all the tested strains were obtained as described above. Actin staining was conducted by previously described (Zhu et al. 2016). All these different samples were transferred to microscope slides and were observed by confocal microscopy (Carl Zeiss LSM 510), using 516-nm and 560 to $615-\mathrm{nm}$ bands as excitation wavelength. Quantitative images were analyzed using the LSM 510 software (version 2.8) and were then processed using Adobe Photoshop 9.0. This experiment was operated at least three times.

\section{Virulence test and microscopic observation.}

Zoospores were induced to produce from the silenced, overexpressed, and control strains as previously described (Fu et al. 2015). The pepper leaves growing at the fifth- to sixth-leaf stage were placed in Petri dishes containing $1.5 \%$ (wt/vol) water agar. Each leaf was spot-inoculated with a zoospore suspension of $2.0 \mu \mathrm{l}\left(1 \times 10^{5}\right.$ zoospores per milliliter $)$ from each strain and were then placed under dark conditions at $25^{\circ} \mathrm{C}$. SD33 and CK were used as controls. Photographs of leaf necrosis were taken at 3 days after incubation. Lesion sizes of were estimated at $5 \mathrm{dpi}$ and their mean areas were also calculated. Mean area differences of 14 inoculation leaves were evaluated statistically by Student's $t$ test. Bars represent the mean \pm standard error from 14 leaves $(P<0.05)$.

In order to observe the germination and growth of cysts in the lesions by microscopy, we inoculated pepper leaves with a mixture of zoospores and mycelia. Negative controls were inoculated with distilled water. Excised lesions of pepper leaf were stained with trypan blue (Zhu et al. 2016). Germ tube production and measurements were made at 6,12 , or $24 \mathrm{hpi}$. In order to visualize the hyphae invasion in the inoculated pepper leaves, tissues of the leaves at 24 hpi were stained with trypan blue (Dong et al. 2011) and were imaged with an Olympus BX53 microscope. Other images were obtained with a digital camera. Each experiment was tested three times from at least 14 pepper leaves.

\section{Statistical analysis.}

Systat 12 was used for data analysis. Briefly, the data were subjected to one-way analysis of variance. Student's $t$ test was used for two means and Duncan's multiple range test of least significant difference was used for more than two means $(P=0.05$ or $P=0.01)$.

\section{ACKNOWLEDGMENTS}

We thank K. Lamour for kindly uploading the $P$. capsici genome sequence to the JGI IMG/M database. We thank B. M. Tyler who contributed many valuable suggestions.

\section{AUTHOR-RECOMMENDED INTERNET RESOURCE}

JGI IMG/M database: http://img.jgi.doe.gov/cgi-bin/w/main.cgi

\section{LITERATURE CITED}

Addinall, S. G., Cao, C., and Lutkenhaus, J. 1997. Temperature shift experiments with an ftsZ84(Ts) strain reveal rapid dynamics of FtsZ localization and indicate that the $\mathrm{Z}$ ring is required throughout septation and cannot reoccupy division sites once constriction has initiated. J. Bacteriol. 179:4277-4284.

Ah-Fong, A. M., Bormann-Chung, C. A., and Judelson, H. S. 2008. Optimization of transgene-mediated silencing in Phytophthora infestans and its association with small-interfering RNAs. Fungal Genet. Biol. 45: 1197-1205.

Anderson, D. E., Gueiros-Filho, F. J., and Erickson, H. P. 2004. Assembly dynamics of FtsZ rings in Bacillus subtilis and Escherichia coli and effects of FtsZ-regulating proteins. J. Bacteriol. 186:5775-5781.

Araki, Y., Takio, S., Ono, K., and Takano, H. 2003. Two types of plastid ftsZ genes in the liverwort Marchantia polymorpha. Protoplasma 221: 163-173.

Arimura, S., and Tsutsumi, N. 2002. A dynamin-like protein (ADL2b), rather than FtsZ, is involved in Arabidopsis mitochondrial division. Proc. Natl. Acad. Sci. U.S.A. 99:5727-5731.

Bachewich, C., and Heath, I. B. 1998. Radial F-actin arrays precede new hypha formation in Saprolegnia: Implications for establishing polar growth and regulating tip morphogenesis. J. Cell Sci. 111: 2005-2016.

Baldauf, S. L., Roger, A. J., Wenk-Siefert, I., and Doolittle, W. F. 2000. A kingdom-level phylogeny of eukaryotes based on combined protein data. Science 290:972-977.

Beech, P. L., Nheu, T., Schultz, T., Herbert, S., Lithgow, T., Gilson, P. R., and McFadden, G. I. 2000. Mitochondrial FtsZ in a chromophyte alga. Science 287:1276-1279.

Ben-Yehuda, S., and Losick, R. 2002. Asymmetric cell division in B. subtilis involves a spiral-like intermediate of the cytokinetic protein FtsZ. Cell 109:257-266.

Bi, E., and Lutkenhaus, J. 1992. Isolation and characterization of ftsZ alleles that affect septal morphology. J. Bacteriol. 174:5414-5423.

Bi, E. F., and Lutkenhaus, J. 1991. FtsZ ring structure associated with division in Escherichia coli. Nature 354:161-164.

Chen, C., MacCready, J. S., Ducat, D. C., and Osteryoung, K. W. 2018. The molecular machinery of chloroplast division. Plant Physiol. 176: 138-151.

Colletti, K. S., Tattersall, E. A., Pyke, K. A., Froelich, J. E., Stokes, K. D., and Osteryoung, K. W. 2000. A homologue of the bacterial cell division site-determining factor MinD mediates placement of the chloroplast division apparatus. Curr. Biol. 10:507-516.

Deora, A., Hashidoko, Y., and Tahara, S. 2008. Actin filaments predominate in morphogenic cell stages, whereas plaques predominate in nonmorphogenic cell stages in Peronosporomycetes. Mycol. Res. 112: 868-882.

Din, N., Quardokus, E. M., Sackett, M. J., and Brun, Y. V. 1998. Dominant Cterminal deletions of FtsZ that affect its ability to localize in Caulobacter and its interaction with FtsA. Mol. Microbiol. 27:1051-1063.

Dong, S., Yin, W., Kong, G., Yang, X., Qutob, D., Chen, Q., Kale, S. D., Sui, Y., Zhang, Z., Dou, D., Zheng, X., Gijzen, M., Tyler, B. M., and Wang, Y. 2011. Phytophthora sojae avirulence effector Avr3b is a secreted NADH and ADP-ribose pyrophosphorylase that modulates plant immunity. PLoS Pathog. 7:e1002353.

Erickson, H. P. 1997. FtsZ, a tubulin homologue in prokaryote cell division. Trends Cell Biol. 7:362-367.

Erickson, H. P., Anderson, D. E., and Osawa, M. 2010. FtsZ in bacterial cytokinesis: Cytoskeleton and force generator all in one. Microbiol. Mol. Biol. Rev. 74:504-528.

Erwin, D. C., and Ribeiro, O. K. 1996. Phytophthora Diseases Worldwide. American Phytopathological Society Press, St. Paul, MN, U.S.A.

Esser, C., Ahmadinejad, N., Wiegand, C., Rotte, C., Sebastiani, F., GeliusDietrich, G., Henze, K., Kretschmann, E., Richly, E., Leister, D., Bryant, D., Steel, M. A., Lockhart, P. J., Penny, D., and Martin, W. 2004. A genome phylogeny for mitochondria among $\alpha$-proteobacteria and a predominantly eubacterial ancestry of yeast nuclear genes. Mol. Biol. Evol. 21:1643-1660.

Fabro, G., Steinbrenner, J., Coates, M., Ishaque, N., Baxter, L., Studholme, D. J., Körner, E., Allen, R. L., Piquerez, S. J., Rougon-Cardoso, A., Greenshields, D., Lei, R., Badel, J. L., Caillaud, M. C., Sohn, K. H., Van den Ackerveken, G., Parker, J. E., Beynon, J., and Jones, J. D. 2011. Multiple candidate effectors from the oomycete pathogen Hyaloperonospora arabidopsidis suppress host plant immunity. PLoS Pathog. 7: e1002348.

Fu, L., Zhu, C., Ding, X., Yang, X., Morris, P. F., Tyler, B. M., and Zhang, X. 2015. Characterization of cell-death-inducing members of the pectate lyase gene family in Phytophthora capsici and their contributions to infection of pepper. Mol. Plant-Microbe Interact. 28:766-775.

Gilson, P. R., and Beech, P. L. 2001. Cell division protein FtsZ: Running rings around bacteria, chloroplasts and mitochondria. Res. Microbiol. 152:3-10. 
Gilson, P. R., Yu, X. C., Hereld, D., Barth, C., Savage, A., Kiefel, B. R., Lay, S., Fisher, P. R., Margolin, W., and Beech, P. L. 2003. Two Dictyostelium orthologs of the prokaryotic cell division protein FtsZ localize to mitochondria and are required for the maintenance of normal mitochondrial morphology. Eukaryot. Cell 2:1315-1326.

Gregory, J. A., Becker, E. C., and Pogliano, K. 2008. Bacillus subtilis MinC destabilizes FtsZ-rings at new cell poles and contributes to the timing of cell division. Genes Dev. 22:3475-3488.

Hale, C. A., Rhee, A. C., and de Boer, P. A. 2000. ZipA-induced bundling of FtsZ polymers mediated by an interaction between $\mathrm{C}$-terminal domains. J. Bacteriol. 182:5153-5166.

Hu, Z., Mukherjee, A., Pichoff, S., and Lutkenhaus, J. 1999. The MinC component of the division site selection system in Escherichia coli interacts with FtsZ to prevent polymerization. Proc. Natl. Acad. Sci. U.S.A. 96:14819-14824.

Hua, C., Meijer, H. J. G., de Keijzer, J., Zhao, W., Wang, Y., and Govers, F. 2013. GK4, a G-protein-coupled receptor with a phosphatidylinositol phosphate kinase domain in Phytophthora infestans, is involved in sporangia development and virulence. Mol. Microbiol. 88: 352-370.

Johnson, J. E., Lackner, L. L., and de Boer, P. A. 2002. Targeting of (D) MinC/MinD and (D)MinC/DicB complexes to septal rings in Escherichia coli suggests a multistep mechanism for MinCmediated destruction of nascent FtsZ rings. J. Bacteriol. 184: 2951-2962.

Judelson, H. S. 2017. Metabolic diversity and novelties in the oomycetes. Annu. Rev. Microbiol. 71:21-39.

Judelson, H. S., and Blanco, F. A. 2005. The spores of Phytophthora: Weapons of the plant destroyer. Nat. Rev. Microbiol. 3:47-58.

Ketelaar, T., Meijer, H. J., Spiekerman, M., Weide, R., and Govers, F. 2012. Effects of latrunculin B on the actin cytoskeleton and hyphal growth in Phytophthora infestans. Fungal Genet. Biol. 49:1014-1022.

Kiefel, B. R., Gilson, P. R., and Beech, P. L. 2004. Diverse eukaryotes have retained mitochondrial homologues of the bacterial division protein FtsZ. Protist 155:105-115.

Kiessling, J., Kruse, S., Rensing, S. A., Harter, K., Decker, E. L., and Reski, R. 2000. Visualization of a cytoskeleton-like FtsZ network in chloroplasts. J. Cell Biol. 151:945-950.

Kots, K., Meijer, H. J., Bouwmeester, K., Govers, F., and Ketelaar, T. 2017. Filamentous actin accumulates during plant cell penetration and cell wall plug formation in Phytophthora infestans. Cell. Mol. Life Sci. 74: 909-920.

Lamour, K. H., Stam, R., Jupe, J., and Huitema, E. 2012. The oomycete broad-host-range pathogen Phytophthora capsici. Mol. Plant Pathol. 13: 329-337.

Latijnhouwers, M., de Wit, P. J., and Govers, F. 2003. Oomycetes and fungi: Similar weaponry to attack plants. Trends Microbiol. 11:462-469.

Leger, M. M., Petrů, M., Žárský, V., Eme, L., Vlček, Č., Harding, T., Lang, B. F., Eliáš, M., Doležal, P., and Roger, A. J. 2015. An ancestral bacterial division system is widespread in eukaryotic mitochondria. Proc. Natl. Acad. Sci. U.S.A. 112:10239-10246.

Levin, P. A., Shim, J. J., and Grossman, A. D. 1998. Effect of minCD on FtsZ ring position and polar septation in Bacillus subtilis. J. Bacteriol. 180:6048-6051.

Liu, H., and Naismith, J. H. 2009. A simple and efficient expression and purification system using two newly constructed vectors. Protein Expr. Purif. 63:102-111.

Ma, X., Ehrhardt, D. W., and Margolin, W. 1996. Colocalization of cell division proteins FtsZ and FtsA to cytoskeletal structures in living Escherichia coli cells by using green fluorescent protein. Proc. Natl. Acad. Sci. U.S.A. 93:12998-13003.

Ma, X., and Margolin, W. 1999. Genetic and functional analyses of the conserved C-terminal core domain of Escherichia coli FtsZ. J. Bacteriol. 181:7531-7544.

Maier, J. A., Ragozin, S., and Jeltsch, A. 2015. Identification, cloning and heterologous expression of active [NiFe]-hydrogenase 2 from Citrobacter sp. SG in Escherichia coli. J. Biotechnol. 199: $1-8$

Matsuzaki, M., Misumi, O., Shin-I, T., Maruyama, S., Takahara, M., Miyagishima, S. Y., Mori, T., Nishida, K., Yagisawa, F., Nishida, K., Yoshida, Y., Nishimura, Y., Nakao, S., Kobayashi, T., Momoyama, Y., Higashiyama, T., Minoda, A., Sano, M., Nomoto, H., Oishi, K., Hayashi, H., Ohta, F., Nishizaka, S., Haga, S., Miura, S., Morishita, T., Kabeya, Y., Terasawa, K., Suzuki, Y., Ishii, Y., Asakawa, S., Takano, H., Ohta, N., Kuroiwa, H., Tanaka, K., Shimizu, N., Sugano, S., Sato, N., Nozaki, H., Ogasawara, N., Kohara, Y., and Kuroiwa, T. 2004. Genome sequence of the ultrasmall unicellular red alga Cyanidioschyzon merolae 10D. Nature 428:653-657.
McLeod, A., Fry, B. A., Zuluaga, A. P., Myers, K. L., and Fry, W. E. 2008. Toward improvements of oomycete transformation protocols. J. Eukaryot. Microbiol. 55:103-109.

Meijer, H. J., Hua, C., Kots, K., Ketelaar, T., and Govers, F. 2014. Actin dynamics in Phytophthora infestans; rapidly reorganizing cables and immobile, long-lived plaques. Cell. Microbiol. 16:948-961.

Miyagishima, S. Y., Nozaki, H., Nishida, K., Nishida, K., Matsuzaki, M., and Kuroiwa, T. 2004. Two types of FtsZ proteins in mitochondria and red-lineage chloroplasts: The duplication of FtsZ is implicated in endosymbiosis. J. Mol. Evol. 58:291-303.

Moore, A. S., and Holzbaur, E. L. F. 2018. Mitochondrial-cytoskeletal interactions: Dynamic associations that facilitate network function and remodeling. Curr. Opin. Physiol. 3:94-100.

Osteryoung, K. W., and McAndrew, R. S. 2001. The plastid division machine. Annu. Rev. Plant Physiol. Plant Mol. Biol. 52:315-333.

Osteryoung, K. W., Stokes, K. D., Rutherford, S. M., Percival, A. L., and Lee, W. Y. 1998. Chloroplast division in higher plants requires members of two functionally divergent gene families with homology to bacterial ftsZ. Plant Cell 10:1991-2004.

Pfaffl, M. W. 2001. A new mathematical model for relative quantification in real-time RT-PCR. Nucleic Acids Res. 29:e45.

Purkanti, R., and Thattai, M. 2015. Ancient dynamin segments capture early stages of host-mitochondrial integration. Proc. Natl. Acad. Sci. U.S.A. 112:2800-2805.

Randall, T. A., Dwyer, R. A., Huitema, E., Beyer, K., Cvitanich, C., Kelkar, H., Fong, A. M., Gates, K., Roberts, S., Yatzkan, E., Gaffney, T., Law, M. Testa, A., Torto-Alalibo, T., Zhang, M., Zheng, L., Mueller, E., Windass, J., Binder, A., Birch, P. R., Gisi, U., Govers, F., Gow, N. A., Mauch, F., van West, P., Waugh, M. E., Yu, J., Boller, T., Kamoun, S., Lam, S. T., and Judelson, H. S. 2005. Large-scale gene discovery in the oomycete Phytophthora infestans reveals likely components of phytopathogenicity shared with true fungi. Mol. Plant-Microbe Interact. 18:229-243.

Sambrook, J., and Russell, D. W. 2001. Molecular Cloning: A Laboratory Manual, 3rd Ed. Cold Spring Harbor Laboratory Press, Cold Spring Harbor, NY.

Sheahan, M. B., McCurdy, D. W., and Rose, R. J. 2005. Mitochondria as a connected population: Ensuring continuity of the mitochondrial genome during plant cell dedifferentiation through massive mitochondrial fusion. Plant J. 44:744-755.

Stokes, K. D., and Osteryoung, K. W. 2003. Early divergence of the FtsZ1 and FtsZ2 plastid division gene families in photosynthetic eukaryotes. Gene 320:97-108.

Suei, S., and Garrill, A. 2008. An F-actin-depleted zone is present at the hyphal tip of invasive hyphae of Neurospora crassa. Protoplasma 232: 165-172.

Takahara, M., Takahashi, H., Matsunaga, S., Miyagishima, S., Takano, H., Sakai, A., Kawano, S., and Kuroiwa, T. 2000. A putative mitochondrial $\mathrm{ftsZ}$ gene is present in the unicellular primitive red alga Cyanidioschyzon merolae. Mol. Gen. Genet. 264:452-460.

Temperli, E., Roos, U. P., and Hohl, H. R. 1990. Actin and tubulin cytoskeletons in germlings of the oomycete fungus Phytophthora infestans. Eur. J. Cell Biol. 53:75-88.

TerBush, A. D., Yoshida, Y., and Osteryoung, K. W. 2013. FtsZ in chloroplast division: Structure, function and evolution. Curr. Opin. Cell Biol. 25:461-470.

Thompson, J. D., Gibson, T. J., Plewniak, F., Jeanmougin, F., and Higgins, D. G. 1997. The CLUSTAL_X windows interface: Flexible strategies for multiple sequence alignment aided by quality analysis tools. Nucleic Acids Res. 25:4876-4882.

Turmel, M., Otis, C., and Lemieux, C. 1999. The complete chloroplast DNA sequence of the green alga Nephroselmis olivacea: Insights into the architecture of ancestral chloroplast genomes. Proc. Natl. Acad. Sci. U.S.A. 96:10248-10253.

Tyler, B. M., Forster, H., and Coffey, M. D. 1995. Inheritance of avirulence factors and restriction fragment length polymorphism markers in outcrosses of the oomycete Phytophthora sojae. Mol. Plant-Microbe Interact. 8:515-523.

Tyler, B. M., Tripathy, S., Zhang, X., Dehal, P., Jiang, R. H., Aerts, A., Arredondo, F. D., Baxter, L., Bensasson, D., Beynon, J. L., Chapman, J., Damasceno, C. M., Dorrance, A. E., Dou, D., Dickerman, A. W., Dubchak, I. L., Garbelotto, M., Gijzen, M., Gordon, S. G., Govers, F., Grunwald, N. J., Huang, W., Ivors, K. L., Jones, R. W., Kamoun, S., Krampis, K., Lamour, K. H., Lee, M. K., McDonald, W. H., Medina, M., Meijer, H. J., Nordberg, E. K., Maclean, D. J., Ospina-Giraldo, M. D., Morris, P. F., Phuntumart, V., Putnam, N. H., Rash, S., Rose, J. K., Sakihama, Y., Salamov, A. A., Savidor, A., Scheuring, C. F., Smith, B. M., Sobral, B. W., Terry, A., Torto-Alalibo, T. A., Win, J., Xu, Z., Zhang, H., Grigoriev, I. V., Rokhsar, D. S., and Boore, J. L. 2006. Phytophthora genome sequences uncover evolutionary origins and mechanisms of pathogenesis. Science 313:1261-1266. 
Vitha, S., McAndrew, R. S., and Osteryoung, K. W. 2001. FtsZ ring formation at the chloroplast division site in plants. J. Cell Biol. 153:111-120.

Walker, C. A., and van West, P. 2007. Zoospore development in the oomycetes. Fungal Biol. Rev. 21:10-18.

Wang, H., Zhang, K., Zhu, J., Song, W., Zhao, L., and Zhang, X. 2013. Structure reveals regulatory mechanisms of a MaoC-like hydratase from Phytophthora capsici involved in biosynthesis of polyhydroxyalkanoates (PHAs). PLoS One 8:e80024.

Ward, J. E., Jr., and Lutkenhaus, J. 1985. Overproduction of FtsZ induces minicell formation in E. coli. Cell 42:941-949.

Westermann, B. 2010. Mitochondrial fusion and fission in cell life and death. Nat. Rev. Mol. Cell Biol. 11:872-884.

Xiao, J., and Goley, E. D. 2016. Redefining the roles of the FtsZ-ring in bacterial cytokinesis. Curr. Opin. Microbiol. 34:90-96

Yan, H. Z., and Liou, R. F. 2006. Selection of internal control genes for realtime quantitative RT-PCR assays in the oomycete plant pathogen Phytophthora parasitica. Fungal Genet. Biol. 43:430-438.

Yang, Y., Sage, T. L., Liu, Y., Ahmad, T. R., Marshall, W. F., Shiu, S. H., Froehlich, J. E., Imre, K. M., and Osteryoung, K. W. 2011. CLUMPED
CHLOROPLASTS 1 is required for plastid separation in Arabidopsis. Proc. Natl. Acad. Sci. U.S.A. 108:18530-18535.

Yee, L. N., Chuah, J. A., Chong, M. L., Phang, L. Y., Raha, A. R., Sudesh, K., and Hassan, M. A. 2012. Molecular characterisation of phaCAB from Comamonas sp. EB172 for functional expression in Escherichia coli JM109. Microbiol. Res. 167:550-557.

Zaremba-Niedzwiedzka, K., Caceres, E. F., Saw, J. H., Bäckström, D., Juzokaite, L., Vancaester, E., Seitz, K. W., Anantharaman, K., Starnawski, P., Kjeldsen, K. U., Stott, M. B., Nunoura, T., Banfield, J. F., Schramm, A., Baker, B. J., Spang, A., and Ettema, T. J. 2017. Asgard archaea illuminate the origin of eukaryotic cellular complexity. Nature 541:353-358.

Zhu, C., Yang, X., Lv, R., Li, Z., Ding, X., Tyler, B. M., and Zhang, X. 2016. Phytophthora capsici homologue of the cell cycle regulator SDA1 is required for sporangial morphology, mycelial growth and plant infection. Mol. Plant Pathol. 17:369-387.

Zottini, M., Barizza, E., Bastianelli, F., Carimi, F., and Lo Schiavo, F. 2006 Growth and senescence of Medicago truncatula cultured cells are associated with characteristic mitochondrial morphology. New Phytol. 172:239-247. 